\title{
Second Order Optimality Conditions for Weak and Strong Local Solutions of Parabolic Optimal Control Problems
}

\author{
Eduardo Casas · Fredi Tröltzsch \\ Dedicated to Prof. Dr. Eberhard Zeidler on the occasion of his 75th birthday
}

the date of receipt and acceptance should be inserted later

\begin{abstract}
Second order sufficient optimality conditions are considered for a simplified class of semilinear parabolic equations with quadratic objective functional including distributed and terminal observation. Main emphasis is laid on problems where the objective functional does not include a Tikhonov regularization term. Here, standard second order conditions cannot be expected to hold. For this case, new second order conditions are established that are based on different types of critical cone. Depending on the choice of this cone, the second order conditions are sufficient for local minima that are weak or strong in the sense of calculus of variations.
\end{abstract}

Keywords Optimal control, parabolic equation, semilinear equation, second order optimality conditions, weak local minimum, strong local minimum

Mathematics Subject Classification (2000) 49J20, 49K20

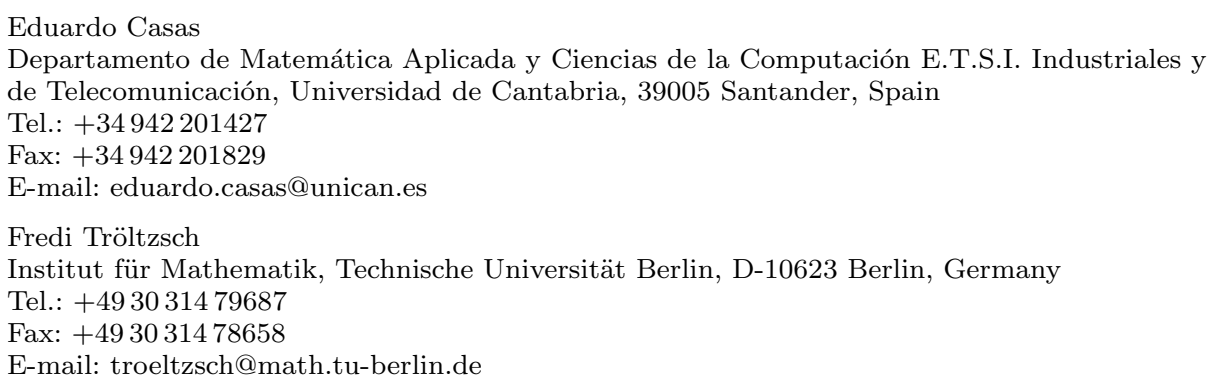




\section{Introduction}

In this paper, we survey second-order optimality conditions for the following slightly simplified class of optimal control problems:

$$
\begin{array}{cl}
\text { Minimize } \frac{1}{2} \int_{Q}\left(y(x, t)-y_{Q}(x, t)\right)^{2} d x d t+\frac{\gamma}{2} \int_{\Omega}\left(y(x, T)-y_{T}(x)\right)^{2} d x \\
+\frac{\nu}{2} \int_{Q} u^{2}(x, t) d x d t
\end{array}
$$

subject to the parabolic state equation

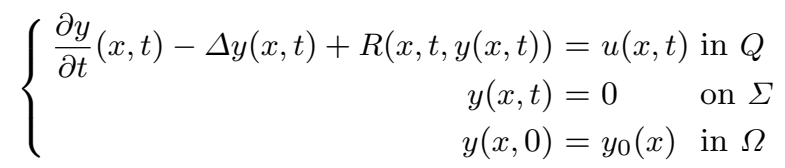

and to the pointwise control constraints

$$
a \leq u(x, t) \leq b \quad \text { for almost all }(x, t) \in Q .
$$

Here, $\Omega \subset \mathbb{R}^{N}, N \in \mathbb{N}$, is a bounded Lipschitz domain with boundary $\Gamma$; $T>0$ is a fixed terminal time and we set $Q=\Omega \times(0, T)$, and $\Sigma=\Gamma \times(0, T)$.

In the optimal control problem, constants $\nu \geq 0, \gamma \geq 0,-\infty<a<b<+\infty$ and desired state functions $y_{Q} \in L^{p}(Q)$ with $p>N / 2+1, y_{T} \in L^{\infty}(\Omega)$, are given.

The function $y$, the state of the control system, is defined as weak solution of (1.2), while the control function $u$ is assumed to be bounded and measurable. The nonlinearity $R: Q \times \mathbb{R} \rightarrow \mathbb{R}$ ("reaction term") is assumed to be a Carathéodory function of class $C^{2}$ with respect to the second variable and satisfies the following assumptions

$$
\begin{aligned}
& \left\{\begin{array}{l}
R(\cdot, \cdot, 0) \in L^{p}(Q), p>N / 2+1, \text { and } \\
\exists C_{R} \in \mathbb{R} \text { such that } \frac{\partial R}{\partial y}(x, t, y) \geq C_{R} \text { for a.a. }(x, t) \in Q \text { and } \forall y \in \mathbb{R},
\end{array}\right. \\
& \left\{\begin{array}{l}
\forall M>0 \exists C_{M}>0 \text { such that } \\
\left|\frac{\partial^{j} R}{\partial y^{j}}(x, t, y)\right| \leq C_{M} \text { for a.a. }(x, t) \in Q, \forall|y| \leq M \text { and } j=1,2,
\end{array}\right. \\
& \left\{\begin{array}{l}
\forall \rho>0 \exists \varepsilon>0 \text { such that } \\
\left|\frac{\partial^{2} R}{\partial y^{2}}\left(x, t, y_{2}\right)-\frac{\partial^{2} R}{\partial y^{2}}\left(x, t, y_{1}\right)\right|<\rho \quad \forall(x, t) \in Q \text { and }\left|y_{2}-y_{1}\right|<\varepsilon .
\end{array}\right.
\end{aligned}
$$

These assumptions include in particular functions $R: \mathbb{R} \longrightarrow \mathbb{R}$ of class $C^{2}$ that are monotone non-decreasing, a standard case in the optimal control of semilinear parabolic equations. Moreover, polynomials of odd order,

$$
R(y)=a_{k} y^{k}+\ldots+a_{1} y+a_{0}
$$

with $k=2 n-1, n \in \mathbb{N}$, and $a_{k}>0$ are allowed. Then $R^{\prime}$ is an even order polynomial and hence bounded from below. Highly nonlinear functions, as $R(y)=\exp (y)$, can be also considered. Further, $y_{0}$ is a given initial state belonging to $L^{\infty}(\Omega)$. 
We discuss second-order conditions that are sufficient for local optimality of stationary solutions of the problem. Stationary solutions are pairs $(\bar{y}, \bar{u})$ that satisfy the first-order necessary optimality conditions of the control problem (1.1)-(1.3). For non-vanishing Tikhonov regularization parameter $\nu>0$, associated results are known since years, we refer to our recent survey paper [12] and the references therein.

Our main interest, however, is the case $\nu=0$, where the objective functional in (1.1) does not contain the last integral that is often called a Tikhonov regularization term; see Corollaries 3 and 4 . We became interested in this degenerate case by numerical observations: The numerical solution method of optimal control problems for the more general FitzHugh-Nagumo system, a well known system of mathematical physics that includes a second linear partial differential equation, turned out to be surprisingly stable for very small regularization parameters $\nu>0$. Eventually, this observation brought us to the ques tion, whether we can prove stability of locally optimal solutions as $\nu \searrow 0$.

To answer this question, we developed second-order sufficient optimality conditions for local solutions that are strong in the sense of calculus of variations and also work for the degenerate case $\nu=0$. Our first associated result is presented in [10]. It is valid for the FitzHugh-Nagumo system and can even be applied to sparse optimal controls, where the objective functional includes in addition the $L^{1}(Q)$-norm of the control function.

To our best knowledge, the first result on strong local minima in PDE control was recently obtained in [1] for the case of semilinear elliptic equations. Moreover, an unknown referee called our attention to the preprint [2] on a parabolic control problem.

Although our results are similar to the ones of [1], [2], they are more general than those of [1], [2]. In particular, we admit the case of a vanishing Tikhonov regularization parameter $\nu$, while $\nu>0$ is required in [1], [2].

In our paper, we introduce a new extended cone of critical directions $E_{\bar{u}}^{\tau}$ prior to Theorem 9 . This cone can be used to deal with the issue of stability for vanishing Tikhonov regularization parameter $\nu$, cf. more detailed remarks in the introduction to Section 3.

On the one hand, the results of [10] are more general than the results we will present in this paper, because they include a nondifferentiable sparsity term. On the other hand, the reaction term $R$ was assumed to be a special third-order polynomial and the domain $\Omega$ was assumed to have a dimension not larger than three. Moreover, due to the more complicated form of the FitzHugh-Nagumo system, the analysis in [10] is very technical.

Therefore, we present our theory of second-order conditions here for a simpler equation but with more general nonlinearity $R$ and for all dimensions $N \in \mathbb{N}$.

\section{Well posedness of state equation and control problem}

We begin our analysis by recalling known results on the solvability of the state equation (1.2). We discuss existence and uniqueness of the state $y$ for given $u$ 
and prove differentiability properties of the control-to-state mapping $G: u \mapsto y$. Adopting a standard notation we set

$$
W(0, T)=\left\{y \in L^{2}\left(0, T ; H_{0}^{1}(\Omega)\right): \frac{\partial y}{\partial t} \in L^{2}\left(0, T ; H^{-1}(\Omega)\right)\right\} .
$$

For the convenience of the reader we recall that a function $y \in W(0, T) \cap L^{\infty}(Q)$ is said to be a weak solution of (1.2), if

$$
\int_{Q}\left\{-y \frac{\partial v}{\partial t}+\nabla y \cdot \nabla v+R(\cdot, \cdot, y) v\right\} d x d t=\int_{Q} u v d x d t+\int_{\Omega} y_{0} v(\cdot, 0) d x
$$

holds for all $v \in H^{1}\left(0, T ; H_{0}^{1}(\Omega)\right)$ such that $v(\cdot, T)=0$.

The following theorem can be shown in the same way as [4, Theorem 1].

Theorem 1 Under our assumptions, for all $u \in L^{p}(Q)$ with $p>N / 2+1$, the equation (1.2) has a unique weak solution $y_{u} \in W(0, T) \cap L^{\infty}(Q)$. There exists a constant $C$ independent of $u$ such that

$$
\left\|y_{u}\right\|_{L^{\infty}(Q)}+\left\|y_{u}\right\|_{W(0, T)} \leq C\left(\|u\|_{L^{p}(Q)}+\left\|y_{0}\right\|_{L^{\infty}(Q)}+\|R(\cdot, \cdot, 0)\|_{L^{p}(Q)}\right) .
$$

and we have $y_{u} \in C(\bar{\Omega} \times(0, T])$. If $y_{0}$ is in addition continuous in $\bar{\Omega}$, then $y_{u}$ belongs to $C(\bar{\Omega} \times[0, T])$.

The main idea of the proof is the use of the substitution $y(x, t)=e^{\lambda t} v(x, t)$ with sufficiently large parameter $\lambda$, cf. [4]. In this way, an equation with monotone nonlinearity is obtained where the known results on existence, uniqueness, and regularity [16, Theorem 5.5] or in [5] can be applied.

Following [11], cf. also [9], we introduce the control-to-state mapping $G: L^{p}(Q)$ $\longrightarrow W(0, T) \cap L^{\infty}(Q), p>N / 2+1$, defined by $G(u)=y_{u}$. Then the following differentiability properties can be proved, see [4] or the more general version $[9$, Theorem 2.2].

Theorem 2 (Differentiability of the control-to-state mapping) The mapping $G$ is of class $C^{2}$. The derivative $\eta_{v}:=G^{\prime}(u) v$ is equal to the function $\eta$ that solves the linearized equation

$$
\left\{\begin{aligned}
\frac{\partial \eta}{\partial t}-\Delta \eta+\frac{\partial R}{\partial y}\left(x, t, y_{u}\right) \eta & =v & \text { in } Q \\
\eta & =0 & \text { on } \Sigma \\
\eta(x, 0) & =0 & \text { in } \Omega .
\end{aligned}\right.
$$

The second derivative $\omega_{v_{1}, v_{2}}:=G^{\prime \prime}(u)\left[v_{1}, v_{2}\right]$ in the directions $v_{1}, v_{2} \in L^{p}(Q)$ is given by the solution $\omega$ of the equation

$$
\left\{\begin{aligned}
\frac{\partial \omega}{\partial t}-\Delta \omega+\frac{\partial R}{\partial y}\left(x, t, y_{u}\right) \omega & =-\frac{\partial^{2} R}{\partial y^{2}}\left(x, t, y_{u}\right) \eta_{v_{1}} \eta_{v_{2}} & & \text { in } Q \\
\omega & =0 & & \text { on } \Sigma \\
\omega(x, 0) & =0 & & \text { in } \Omega,
\end{aligned}\right.
$$

where $\eta_{v_{i}}=G^{\prime}(u) v_{i}, i=1,2$. 
Remark 1 The operator $G$ is not differentiable from $L^{2}(Q)$ to $W(0, T)$. However, $G^{\prime}(u)$ and $G^{\prime \prime}(u)$ can be extended to continuous linear and bilinear mappings from $L^{2}(Q)$ to $W(0, T)$, for any $u \in L^{p}(Q)$ with $p>N / 2+1$.

Since $y_{u}$ is uniquely determined by $u$, we can formulate the optimal control problem (1.2)-(1.3) in the following control reduced form:

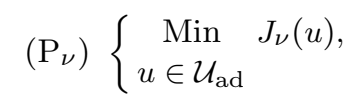

where the (control reduced) objective functional is defined by

$$
\begin{aligned}
J_{\nu}(u):= & \frac{1}{2} \int_{Q}\left(y_{u}(x, t)-y_{Q}(x, t)\right)^{2} d x d t+\frac{\gamma}{2} \int_{\Omega}\left(y_{u}(x, T)-y_{T}(x)\right)^{2} d x \\
& +\frac{\nu}{2} \int_{Q} u^{2}(x, t) d x d t
\end{aligned}
$$

and the set of admissible controls $\mathcal{U}_{\text {ad }}$ is

$$
\mathcal{U}_{\mathrm{ad}}=\left\{u \in L^{\infty}(Q): a \leq u(x, t) \leq b \text { for a.a. }(x, t) \in Q\right\} .
$$

Thanks to Theorems 1 and 2 , it is easy to prove that for all $\nu \geq 0$ the control problem $\left(\mathrm{P}_{\nu}\right)$ has at least one solution $\bar{u}_{\nu}$; see [9, Theorem 3.1]. In view of the differentiability properties of $G$, also $J_{\nu}$ is of class $C^{2}$. In a standard way, the derivatives can be expressed by using an adjoint equation. For given $u \in L^{p}(Q)$, the equation

$$
\left\{\begin{array}{rlrl}
-\frac{\partial \varphi_{u}}{\partial t}-\Delta \varphi_{u}+\frac{\partial R}{\partial y}\left(x, t, y_{u}\right) \varphi_{u} & =y_{u}-y_{Q} & & \text { in } Q \\
\varphi_{u} & =0 & & \text { on } \Sigma \\
\varphi_{u}(x, T) & =\gamma\left(y_{u}(T)-y_{T}\right) & \text { in } \Omega
\end{array}\right.
$$

is called the adjoint equation. Its solution $\varphi_{u}$ is the adjoint state associated with $u$.

Theorem 3 The functional $J_{\nu}: L^{p}(Q) \longrightarrow \mathbb{R}, p>N / 2+1$, is of class $C^{2}$. Its first order derivative is given by

$$
J_{\nu}^{\prime}(u) v=\int_{Q}\left(\varphi_{u}+\nu u\right) v d x d t \quad \forall u, v \in L^{p}(Q),
$$

where $\varphi_{u}$ is the solution in $W(0, T) \cap L^{\infty}(Q)$ of the adjoint equation (2.4). For directions $v_{1}, v_{2} \in L^{p}(Q)$, the second order derivative of $J_{\nu}$ is

$$
\begin{aligned}
J_{\nu}^{\prime \prime}(u)\left[v_{1}, v_{2}\right]= & \int_{Q}\left[1-\frac{\partial^{2} R}{\partial y^{2}}\left(x, t, y_{u}\right) \varphi_{u}\right] \eta_{v_{1}} \eta_{v_{2}} d x d t \\
& +\gamma \int_{\Omega} \eta_{v_{1}}(T) \eta_{v_{2}}(T) d x+\nu \int_{Q} v_{1} v_{2} d x d t .
\end{aligned}
$$

The proof of the existence and uniqueness of the solution $\varphi_{u} \in W(0, T) \cap L^{\infty}(Q)$ of the adjoint system and the formula $(2.5)$ can be found in $[9, \S 3.2]$. The expression of the second derivative follows from the chain rule, (2.2), and (2.4). 


\section{First-Order Necessary Optimality Conditions}

Since the control problem $\left(\mathrm{P}_{\nu}\right)$ is not convex, we consider local minima. In this section we set up the associated first-order necessary optimality conditions and draw some conclusions from the optimality system.

We say that $\bar{u}_{\nu}$ is a local minimum of problem $\left(\mathrm{P}_{\nu}\right)$ in the sense of $L^{p}(Q)$, $1 \leq p \leq+\infty$, if there exists $\varepsilon>0$ such that

$$
J_{\nu}\left(\bar{u}_{\nu}\right) \leq J_{\nu}(u) \quad \forall u \in \mathcal{U}_{\mathrm{ad}} \cap B_{\varepsilon}\left(\bar{u}_{\nu}\right) .
$$

Here, $B_{\varepsilon}\left(\bar{u}_{\nu}\right)$ denotes the $L^{p}(Q)$-ball centered at $\bar{u}_{\nu}$ with radius $\varepsilon$.

The boundedness of $\mathcal{U}_{\mathrm{ad}}$ in $L^{\infty}(Q)$ implies that $\bar{u}_{\nu}$ is a local minimum in the $L^{2}(Q)$ sense if and only if it is a local minimum in the $L^{p}(Q)$ sense for any $1 \leq p<+\infty$. On the other hand, if $\bar{u}_{\nu}$ is a local minimum in the $L^{\infty}(Q)$ sense, then it is a local minimum in the $L^{p}(Q)$ sense for any $1 \leq p \leq+\infty$. Hereafter, local minima will be always understood as local minima in the $L^{2}(Q)$ sense.

Remark 2 Minima of the type (3.1) are, viewed in the sense of calculus of variations, weak local minima. This means that $J_{\nu}\left(\bar{u}_{\nu}\right) \leq J_{\nu}(u)$ is satisfied for all admissible $u$ out of a neighborhood of the control $\bar{u}_{\nu}$.

Notice that the control $u$ is the image of $y_{u}$ of the differential operator $y \mapsto$ $\partial y / \partial t-\Delta y+R(\cdot, \cdot, y)$. Therefore $u$ plays the role of the derivative $y^{\prime}$ of the unknown function $x \mapsto y(x)$ in the classical calculus of variations. In this sense, the neighborhood $B_{\varepsilon}\left(\bar{u}_{\nu}\right)$ is a neighborhood that accounts also for the derivatives of $y$.

Later, we will also investigate conditions for local minima that are strong in the sense of calculus of variations. This means that

$$
J_{\nu}\left(\bar{u}_{\nu}\right) \leq J_{\nu}(u) \quad \forall u \in \mathcal{U}_{\mathrm{ad}} \text { such that }\left\|y_{u}-y_{\bar{u}_{\nu}}\right\|_{L^{\infty}(Q)}<\varepsilon,
$$

no matter how far $u$ is from $\bar{u}_{\nu}$.

For the definition and discussion of weak and strong local minima in the classical calculus of variations, we refer to [15, Chpt. 2] and [18, Sect. 37.4e].

The following first order necessary optimality conditions have to be satisfied by any local minimum of $\left(\mathrm{P}_{\nu}\right)$.

Theorem 4 Let $\bar{u}_{\nu}$ be a local minimum of $\left(\mathrm{P}_{\nu}\right)$, let $\bar{y}_{\nu}$ be the associated state, and let $\bar{\varphi}_{\nu}:=\varphi_{\bar{u}_{\nu}} \in W(0, T) \cap L^{\infty}(Q)$ be the associated adjoint state defined as the unique solution of (2.4) for $u:=\bar{u}_{\nu}$. Then $\bar{u}_{\nu}$ obeys the variational inequality

$$
\int_{Q}\left(\bar{\varphi}_{\nu}+\nu \bar{u}_{\nu}\right)\left(u-\bar{u}_{\nu}\right) d x d t \geq 0 \quad \forall u \in \mathcal{U}_{\mathrm{ad}}
$$

The well-known projection formula below follows by a standard discussion of the variational inequality (3.2). 
Corollary 1 Let $\bar{u}_{\nu}, \bar{\varphi}_{\nu}$ be as in Theorem \& and assume that $\nu>0$. Then

$$
\bar{u}_{\nu}(x, t)=\operatorname{Proj}_{[a, b]}\left(-\frac{1}{\nu} \bar{\varphi}_{\nu}(x, t)\right),
$$

where $\operatorname{Proj}_{[a, b]}: \mathbb{R} \rightarrow[a, b]$ is defined by $\operatorname{Proj}_{[a, b]}(z)=\max \{a, \min \{b, z\}\}$.

From the projection formula above, the regularity $\bar{u}_{\nu} \in L^{2}\left(0, T ; H^{1}(\Omega)\right)$ is obtained.

Corollary 2 Let $\bar{u}_{\nu}$ and $\bar{\varphi}_{\nu}$ be as in Theorem 4 and assume that $\nu=0$. Then the following implications hold,

$$
\left\{\begin{array} { r l } 
{ \overline { u } _ { \nu } ( t , x ) = a } & { \Rightarrow \overline { \varphi } _ { \nu } ( t , x ) \geq 0 , } \\
{ \overline { u } _ { \nu } ( t , x ) = b } & { \Rightarrow \overline { \varphi } _ { \nu } ( t , x ) \leq 0 , } \\
{ a < \overline { u } _ { \nu } ( t , x ) < b } & { \Rightarrow \overline { \varphi } _ { \nu } ( t , x ) = 0 }
\end{array} \text { and } \left\{\begin{array}{l}
\bar{\varphi}_{\nu}(x, t)>0 \Rightarrow \bar{u}_{\nu}(x, t)=a, \\
\bar{\varphi}_{\nu}(x, t)<0 \Rightarrow \bar{u}_{\nu}(x, t)=b .
\end{array}\right.\right.
$$

From the above relations we deduce that the optimal control $\bar{u}_{\nu}$ is bang-bang if the set of points of $Q$ where $\bar{\varphi}_{\nu}$ vanishes has a zero Lebesgue measure.

For these well known results, we refer the reader to [8, Corollary 3.2] and [6, Theorem 3.1] that deal with the cases $\nu>0$ and $\nu=0$, respectively, in a similar situation. Moreover, these implications are extensively explained in [16, p.70] for the elliptic case.

\section{Second Order Optimality Conditions}

Next, we develop the second order analysis for $\left(\mathrm{P}_{\nu}\right)$ and begin with second order necessary conditions. Let a control $\bar{u}_{\nu} \in \mathcal{U}_{\text {ad }}$ fulfill the optimality conditions of Theorem 4 . This means that $\bar{u}_{\nu}$ obeys, along with the adjoint state $\bar{\varphi}_{\nu}$, the variational inequality (3.2). Associated with $\bar{u}_{\nu}$, we introduce the standard cone of critical directions

$$
C \bar{u}_{\nu}=\left\{v \in L^{2}(Q): v \text { obeys the sign conditions (4.1) below and } J_{\nu}^{\prime}\left(\bar{u}_{\nu}\right) v=0\right\},
$$

$$
v(x, t)\left\{\begin{array}{l}
\geq 0 \text { if } \bar{u}_{\nu}(x, t)=a \\
\leq 0 \text { if } \bar{u}_{\nu}(x, t)=b
\end{array}\right.
$$

The next theorem is well known; the reader is referred to [3] or [11] for a general result.

Theorem 5 (Second order necessary condition) The set $C_{\bar{u}_{\nu}}$ is a convex and closed cone in $L^{2}(Q)$. If $\bar{u}_{\nu}$ is locally optimal for $\left(\mathrm{P}_{\nu}\right)$, then

$$
J_{\nu}^{\prime \prime}\left(\bar{u}_{\nu}\right) v^{2} \geq 0 \forall v \in C_{\bar{u}_{\nu}} .
$$


Now we turn over to second order sufficient conditions. Depending on different situations, besides $C_{\bar{u}_{\nu}}$ we will introduce two more cones of critical directions, namely the cones $C_{\bar{u}}^{\tau}$ and $E_{\bar{u}}^{\tau}$.

The cone $C_{\bar{u}}$, considered for the necessary second order conditions, will also appear in the formulation of second order sufficient conditions for $\nu>0$ in Thm. 6 . This is a result on weak local optimality. However, as a corollary, we obtain the surprising result that, in this case, $\bar{u}_{\nu}$ yields even a strong local minimum.

The two other cones are introduced for the case $\nu=0$. The second order conditions based on $E_{\bar{u}}^{\tau}$ guarantee that $\bar{y}$ affords a minimum to the objective functional that is strong in the sense of calculus of variations, cf. Theorem 9 and relation (4.35). This property of strong local optimality cannot be deduced, if we substitute $C_{\bar{u}}^{\tau}$ for $E_{\bar{u}}^{\tau}$.

In Section 5, we shall apply these different second order conditions to proving results on stability of optimal solution with respect to certain perturbations of the data in the optimal control problem.

\subsection{The case $\boldsymbol{\nu}>\mathbf{0}$}

The following properties of $C_{\bar{u}_{\nu}}$ will be needed.

\section{Lemma 1}

(i) If $v \in L^{2}(Q)$ satisfies (4.1), then $\left(\bar{\varphi}_{\nu}+\nu \bar{u}_{\nu}\right) v \geq 0$ holds a.e. in $Q$.

(ii) If $v \in C_{\bar{u}_{\nu}}$, then

$$
\left(\bar{\varphi}_{\nu}+\nu \bar{u}_{\nu}\right) v=0 \text { a.e. in } Q .
$$

(iii) A function $v \in L^{2}(Q)$ belongs to $C_{\bar{u}_{\nu}}$ iff the following properties hold true a.e.,

$$
v(x, t)\left\{\begin{array}{l}
=0 \text { if }\left|\bar{\varphi}_{\nu}(x, t)+\nu \bar{u}_{\nu}(x, t)\right|>0 \\
\geq 0 \text { if } \bar{u}_{\nu}(x, t)=a \\
\leq 0 \text { if } \bar{u}_{\nu}(x, t)=b
\end{array}\right.
$$

Proof Statement (i) is a simple consequence of Corollary 1. Indeed, if $\left(\bar{\varphi}_{\nu}+\right.$ $\left.\nu \bar{u}_{\nu}\right)(x, t)>0$, then we have $\bar{u}_{\nu}(x, t)>-\frac{1}{\nu} \bar{\varphi}_{\nu}(x, t)$. In view of the projection formula for $\bar{u}_{\nu}$, this inequality is possible only if $\bar{u}_{\nu}(x, t)=a$. Then (4.1) implies that $v(x, t) \geq 0$. Analogously, we proceed when $\left(\bar{\varphi}_{\nu}+\nu \bar{u}_{\nu}\right)(x, t)<0$.

Now (ii) follows immediately: If $v \in C_{\bar{u}_{\nu}}$, then $v$ obeys in particular the sign conditions (4.1). By (i), $\left(\bar{\varphi}_{\nu}+\nu \bar{u}_{\nu}\right) v$ is almost everywhere nonnegative. Moreover, $J_{\nu}^{\prime}\left(\bar{u}_{\nu}\right) v=0$ is included in the definition of $C_{\bar{u}_{\nu}}$, hence

$$
0=J_{\nu}^{\prime}\left(\bar{u}_{\nu}\right) v=\int_{Q}\left(\bar{\varphi}_{\nu}+\nu \bar{u}_{\nu}\right) v d x d t
$$

Since the integrand is nonnegative, this can only hold, if (4.2) is true. 
To show (iii), we consider first an element $v \in L^{2}(Q)$ satisfying (4.3). Then $v$ obeys (4.1) and it remains to prove that $J_{\nu}^{\prime}\left(\bar{u}_{\nu}\right) v=0$. This, however, is obvious by the representation $(4.4)$ for $J_{\nu}^{\prime}\left(\bar{u}_{\nu}\right) v$ : Whenever $\left(\bar{\varphi}_{\nu}+\nu \bar{u}_{\nu}\right)(x, t) \neq 0$, then $v(x, t)$ vanishes by (4.3). Conversely, if $v \in C_{\bar{u}_{\nu}}$, then the sign conditions are fulfilled again by definition. The implication $\left(\bar{\varphi}_{\nu}+\nu \bar{u}_{\nu}\right)(x, t) \neq 0 \Rightarrow v(x, t)=0$ follows immediately from (4.2).

Our next auxiliary result is important. The equivalence that is stated in the following lemma is not in general true for infinite-dimensional optimization problems. However, it holds true if the Tikhonov regularization term $\frac{\nu}{2}\|u\|_{L^{2}(Q)}^{2}$ is included in the cost functional with $\nu>0$; see [12] for the proof.

Lemma 2 The following statements are equivalent

(i) $J_{\nu}^{\prime \prime}\left(\bar{u}_{\nu}\right) v^{2}>0 \quad \forall v \in C_{\bar{u}_{\nu}} \backslash\{0\}$.

(ii) There exists $\sigma>0$ such that

$$
J_{\nu}^{\prime \prime}\left(\bar{u}_{\nu}\right) v^{2} \geq \sigma\|v\|_{L^{2}(Q)}^{2} \quad \forall v \in C_{\bar{u}_{\nu}} .
$$

In the case of unconstrained optimization, the coercivity condition (4.5) can be required in the whole space and is sufficient for a local minimum. For a comparison with this case, we refer to [19, Sect. 2.2].

Finally, we recall the following result.

Theorem 6 ( $\nu>0$; Second order sufficient condition, [11]) Assume $\nu>0$ and let $\bar{u}_{\nu} \in \mathcal{U}_{\mathrm{ad}}$, along with the adjoint state $\bar{\varphi}_{\nu}$, satisfy the variational inequality (3.2). Assume also that $J_{\nu}^{\prime \prime}\left(\bar{u}_{\nu}\right) v^{2}>0 \forall v \in C_{\bar{u}_{\nu}} \backslash\{0\}$. Then $\delta>0$ and $\varepsilon>0$ exist such that

$$
J_{\nu}\left(\bar{u}_{\nu}\right)+\frac{\delta}{2}\left\|u-\bar{u}_{\nu}\right\|_{L^{2}(Q)}^{2} \leq J_{\nu}(u) \quad \forall u \in \mathcal{U}_{\mathrm{ad}} \cap B_{\varepsilon}\left(\bar{u}_{\nu}\right),
$$

where $B_{\varepsilon}\left(\bar{u}_{\nu}\right)$ is the $L^{2}(Q)$-ball centered at $\bar{u}_{\nu}$ with radius $\varepsilon$.

Compared with the classical calculus of variations, this result means that $\bar{u}$ affords to $J_{\nu}$ a weak local minimum. However, we are able to prove the surprising fact that the local optimality ensured by Theorem 6 is even strong.

Theorem 7 ( $\nu>0$; Strong local optimality) Let $\bar{u}_{\nu}$ satisfy all assumptions of Theorem 6. Then $\delta^{\prime}>0$ and $\varepsilon^{\prime}>0$ exist such that

$$
J_{\nu}\left(\bar{u}_{\nu}\right)+\frac{\delta^{\prime}}{2}\left\|u-\bar{u}_{\nu}\right\|_{L^{2}(Q)}^{2} \leq J_{\nu}(u) \quad \forall u \in \mathcal{U}_{\mathrm{ad}}:\left\|y_{u}-\bar{y}_{\nu}\right\|_{L^{\infty}(Q)}<\varepsilon^{\prime} .
$$

Proof Assume the contrary, i.e. that (4.7) does not hold for any $\delta^{\prime}$ and $\varepsilon^{\prime}$. Then, for any integer $k \geq 1$, we can find a control $u_{k} \in \mathcal{U}_{\text {ad }}$ with $\left\|y_{u_{k}}-\bar{y}_{\nu}\right\|_{L^{\infty}(Q)}<1 / k$ such that

$$
J_{\nu}\left(u_{k}\right)<J_{\nu}\left(\bar{u}_{\nu}\right)+\frac{1}{2 k}\left\|u_{k}-\bar{u}_{\nu}\right\|_{L^{2}(Q)}^{2} .
$$


We can select a subsequence, denoted in the same way, such that $\left\{u_{k}\right\}_{k \geq 1}$ is weakly* convergent to some $\tilde{u} \in L^{\infty}(Q)$. From the parabolic equation, we have

$$
\partial_{t} y_{k}-\Delta y_{k}=-R\left(x, t, y_{k}\right)+u_{k} .
$$

The right-hand side converges weakly in $L^{p}(Q)$ to $-R\left(x, t, \bar{y}_{\nu}\right)+\tilde{u}$. Therefore, by the weak continuity of the solution mapping for linear parabolic equations, the sequence $\left\{y_{k}\right\}$ converges weakly in $W(0, T)$ to a solution $\tilde{y}$ that satisfies

$$
\partial_{t} \tilde{y}-\Delta \tilde{y}=-R\left(x, t, \bar{y}_{\nu}\right)+\tilde{u} \text {. }
$$

From $y_{k} \rightarrow \tilde{y}$ in $W(0, T)$ and $y_{k} \rightarrow \bar{y}_{\nu}$ in $L^{\infty}(Q)$, we find $\tilde{y}=\bar{y}_{\nu}$ and hence $\tilde{u}=\bar{u}$. In this way, $u_{k} \stackrel{*}{\rightarrow} \bar{u}_{\nu}$ in $L^{\infty}(Q)$ is obtained. In particular, we also have that $u_{k} \rightarrow \bar{u}_{\nu}$ in $L^{2}(Q)$.

By our notation and the convergence $y_{k} \rightarrow \bar{y}_{\nu}$ in $L^{\infty}(Q)$, we have $J_{0}\left(u_{k}\right) \rightarrow$ $J_{0}\left(\bar{u}_{\nu}\right)$; hence we deduce from (4.8)

$$
\frac{\nu}{2}\left\|u_{k}\right\|_{L^{2}(Q)}^{2} \leq \frac{\nu}{2}\left\|\bar{u}_{\nu}\right\|_{L^{2}(Q)}^{2}+J_{0}\left(\bar{u}_{\nu}\right)-J_{0}\left(u_{k}\right)+\frac{1}{2 k}\left\|u_{k}-\bar{u}_{\nu}\right\|_{L^{2}(Q)}^{2} .
$$

Passing to the limit $k \rightarrow \infty$, we arrive at

$$
\frac{\nu}{2}\left\|\bar{u}_{\nu}\right\|_{L^{2}(Q)}^{2} \leq \liminf _{k \rightarrow \infty} \frac{\nu}{2}\left\|u_{k}\right\|_{L^{2}(Q)}^{2} \leq \limsup _{k \rightarrow \infty} \frac{\nu}{2}\left\|u_{k}\right\|_{L^{2}(Q)}^{2}=\frac{\nu}{2}\left\|\bar{u}_{\nu}\right\|_{L^{2}(Q)}^{2} .
$$

This implies that $\left\|u_{k}\right\|_{L^{2}(Q)}^{2} \rightarrow\left\|\bar{u}_{\nu}\right\|_{L^{2}(Q)}^{2}$ holds in addition to the weak convergence. Therefore, the convergence of $\left\{u_{k}\right\}$ to $\bar{u}_{\nu}$ is strong in $L^{2}(Q)$. Consequently, given $\epsilon>0$ such that (4.6) holds, we have that $\left\|u_{k}-\bar{u}_{\nu}\right\|_{L^{2}(Q)}<\varepsilon$ for all $k$ sufficiently large. Then (4.8) contradicts (4.6).

In the framework of optimal control of partial differential equations, the first result about strong local optimality was proved in [1] for the elliptic case.

\subsection{The case $\boldsymbol{\nu}=\mathbf{0}$}

Our main results on second order conditions for $\nu>0$ (except Theorem 7 ) were known since for some years, cf. [11]. In the degenerate case $\nu=0$, we found our results just recently in [10] for the FitzHugh-Nagumo system. Here, we adapt the ideas of [10] to our semilinear parabolic state equation with slightly more general nonlinearity $R$.

To simplify the notation, we will write $(\mathrm{P})$ instead of $\left(\mathrm{P}_{0}\right), J$ instead of $J_{0}$, and $\bar{u}, \bar{y}, \bar{\varphi}$, etc., instead of $\bar{u}_{0}, \bar{y}_{0}, \bar{\varphi}_{0}$.

For general infinite-dimensional optimization problems, the strict positivity of the second derivative of the objective functional on the critical cone is not sufficient for local optimality. An associated example is known from [14]. (The situation is different for our objective functional $J_{\nu}$, if $\nu>0$. Then the second derivative generates a Legendre form (cf. [15, Chpt. 6.2.1]) and we were able to argue as in the preceding section). 
Therefore, we have to consider the well-known extended cone $C_{\bar{u}}^{\tau}$, cf. [13] for the elliptic case or $[16,(5.42)]$ for a parabolic state equation. For given (small) threshold $\tau>0$, we define $C_{\bar{u}}^{\tau}$ as the set of elements $v \in L^{2}(Q)$ satisfying

$$
v(x, t)\left\{\begin{array}{l}
=0 \text { if }|\bar{\varphi}(x, t)| \geq \tau, \\
\geq 0 \text { if } \bar{u}(x, t)=a \\
\leq 0 \text { if } \bar{u}(x, t)=b .
\end{array}\right.
$$

Proposition 1 The extended cone $C_{\bar{u}}^{\tau}$ covers $C_{\bar{u}}$, i.e. $C_{\bar{u}} \subset C_{\bar{u}}^{\tau}$ holds for all $\tau>0$. For every $v \in L^{2}(Q)$ satisfying the sign conditions (4.1), the inequality

$$
J^{\prime}(\bar{u}) v \geq \tau\|v\|_{L^{1}\left(Q_{v}\right)}
$$

is fulfilled, where $Q_{v}$ denotes the set of points $(x, t) \in Q$ such that the first condition of (4.9) is not satisfied by $v(x, t)$.

Proof The inclusion $C_{\bar{u}} \subset C_{\bar{u}}^{\tau}$ is obvious. Let us prove the second claim.

If $\bar{\varphi}(x, t) \geq \tau$, then Corollary 2 implies that $\bar{u}(x, t)=a$ and therefore $v(x, t) \geq 0$, hence $\bar{\varphi}(x, t) v(x, t) \geq \tau|v(x, t)|$. Analogously, we show $\bar{\varphi}(x, t) v(x, t) \geq \tau|v(x, t)|$, if $-\bar{\varphi}(x, t) \leq \tau$. Therefore,

$$
\begin{aligned}
J^{\prime}(\bar{u}) v & =\int_{Q \backslash Q_{v}} \bar{\varphi}(x, t) v(x, t) d x d t+\int_{Q_{v}} \bar{\varphi}(x, t) v(x, t) d x d t \\
& \geq \int_{Q_{v}} \bar{\varphi}(x, t) v(x, t) d x d t \geq \tau \int_{Q_{v}}|v(x, t)| d x d t=\tau\|v\|_{L^{1}\left(Q_{v}\right)} .
\end{aligned}
$$

The nonnegative of the first integral in the inequalities above follows from Lemma $1,(\mathrm{i})$.

Now one might be tempted to formulate the second order sufficient conditions as

$$
\exists \tau>0 \text { and } \exists \sigma>0 \text { such that } J^{\prime \prime}(\bar{u}) v^{2} \geq \sigma\|v\|_{L^{2}(Q)}^{2} \quad \forall v \in C_{\bar{u}}^{\tau} .
$$

Unfortunately, this condition cannot be expected for $\nu=0$, since the Tikhonov regularization term is missing. This term is needed to fulfill the condition above. Actually, the inequality (4.11) holds in a few very exceptional cases; see [6] and [10].

The following second order sufficient condition is adequate:

Theorem 8 Let $\bar{u} \in \mathcal{U}_{\mathrm{ad}}$, along with the adjoint state $\bar{\varphi}=\varphi \bar{u}$, satisfy the variational inequality (3.2). Assume that

$$
\exists \tau>0 \text { and } \exists \sigma>0: J^{\prime \prime}(\bar{u}) v^{2} \geq \sigma\left(\left\|\eta_{v}\right\|_{L^{2}(Q)}^{2}+\gamma\left\|\eta_{v}(T)\right\|_{L^{2}(\Omega)}^{2}\right) \forall v \in C_{\bar{u}}^{\tau},
$$

where $\eta_{v}=G^{\prime}(\bar{u}) v$. Then, there exists $\varepsilon>0$ such that

$$
J(\bar{u})+\frac{\sigma}{16}\left(\left\|\eta_{u-\bar{u}}\right\|_{L^{2}(Q)}^{2}+\gamma\left\|\eta_{u-\bar{u}}(T)\right\|_{L^{2}(\Omega)}^{2}\right) \leq J(u) \quad \forall u \in \mathcal{U}_{\mathrm{ad}} \cap B_{\varepsilon}(\bar{u}),
$$

where $B_{\varepsilon}(\bar{u})$ is the ball of $L^{2}(Q)$ centered at $\bar{u}$ with radius $\varepsilon$. 
The inequality (4.12) of the second order condition in Theorem 4.13 can be motivated by the form of the second order derivative $J^{\prime \prime}(\bar{u})[v, v]$ in Theorem 3: If $\nu=0$, then the $L^{2}(Q)$-norm of $v^{2}$ is missing, while the associated $L^{2}$-norms of $\eta_{v}$ and $\eta_{v}(T)$ are still present.

Before proving this theorem, we derive some auxiliary results.

Lemma 3 Assume that $p>N / 2+1$. Then constants $C_{a, b}, C_{1}, C_{2}, C_{3}$, and $C_{\infty}$ exist such that, for all $u \in \mathcal{U}_{\mathrm{ad}}$, the following estimates are satisfied:

$$
\begin{aligned}
& \left\|y_{u}\right\|_{W(0, T)}+\left\|y_{u}\right\|_{L^{\infty}(Q)}+\left\|\varphi_{u}\right\|_{W(0, T)}+\left\|\varphi_{u}\right\|_{L^{\infty}(Q)} \leq C_{a, b} \\
& \left\|y_{u}-\bar{y}\right\|_{W(0, T)}+\left\|\varphi_{u}-\bar{\varphi}\right\|_{W(0, T)} \leq C_{2}\|u-\bar{u}\|_{L^{2}(Q)} \\
& \left\|\varphi_{u}-\bar{\varphi}\right\|_{L^{\infty}(Q)} \leq C_{\infty}\left\|y_{u}-\bar{y}\right\|_{L^{\infty}(Q)} \leq C_{p}\|u-\bar{u}\|_{L^{p}(Q)} \\
& \left\|\eta_{v}\right\|_{L^{2}(Q)}^{2}+\gamma\left\|\eta_{v}(T)\right\|_{L^{2}(\Omega)}^{2} \leq C_{1}\|v\|_{L^{p}(Q)}\|v\|_{L^{1}(Q)} \quad \forall v \in L^{p}(Q) .
\end{aligned}
$$

Proof The first estimate follows from Theorem 1 and the estimates for the solution of (2.4). To prove the second and third estimates, we we substitute $\left(y_{u}-\bar{y}\right)(x, t)=$ $\mathrm{e}^{\mu t} w(x, t)$ with some $\mu \geq-c_{R}$, see (1.4), and subtract the parabolic equations satisfied by $y_{u}$ and $\bar{y}$. We obtain

$$
\left\{\begin{aligned}
\frac{\partial w}{\partial t}-\Delta w+\left(\mathrm{e}^{-\mu t} \frac{\partial R}{\partial y}\left(x, t, \hat{y}_{u}\right)+\mu\right) w & =\mathrm{e}^{-\mu t}(u-\bar{u}) & & \text { in } Q \\
w & =0 & & \text { on } \Sigma \\
w(x, 0) & =0 & & \text { in } \Omega,
\end{aligned}\right.
$$

where $\hat{y}_{u}(x, t)=\bar{y}(x, t)+\theta(x, t)\left(y_{u}(x, t)-\bar{y}(x, t)\right)$ and $0 \leq \theta(x, t) \leq 1$. In view of (1.4), $\mathrm{e}^{-\mu t} \frac{\partial R}{\partial y}\left(x, t, \hat{y}_{u}\right)+\mu$ is a.e. nonnegative. Now a standard estimate for linear parabolic equations leads to an estimate of the type (4.15) for $\|w\|$. Transforming back by $y_{u}-\bar{y}=\mathrm{e}^{\mu t} w$ delivers the $W(0, T)$-estimate for $y_{u}-\bar{y}$ in (4.15).

To confirm the associated estimate for $\varphi_{u}-\bar{\varphi}$, we consider the difference of the adjoint equations for $\varphi_{u}$ and $\bar{\varphi}$,

$$
\begin{aligned}
& \frac{\partial\left(\varphi_{u}-\bar{\varphi}\right)}{\partial t}-\Delta\left(\varphi_{u}-\bar{\varphi}\right)+\frac{\partial R}{\partial y}(x, t, \bar{y})\left(\varphi_{u}-\bar{\varphi}\right) \\
& =\left(\frac{\partial R}{\partial y}(x, t, \bar{y})-\frac{\partial R}{\partial y}\left(x, t, y_{u}\right)\right)\left(\varphi_{u}-\bar{\varphi}\right)+y_{u}-\bar{y} \text { in } Q, \\
& \left(\varphi_{u}-\bar{\varphi}\right)=0 \text { on } \Sigma, \quad\left(\varphi_{u}-\bar{\varphi}\right)(T)=\gamma\left(y_{u}-\bar{y}\right)(T) \text { in } \Omega .
\end{aligned}
$$

Notice that $\frac{\partial R}{\partial y}(x, t, \bar{y})$ might be negative, hence we consider the transformed difference $\mathrm{e}^{-\mu t}\left(\varphi_{u}-\bar{\varphi}\right)$.

From the estimate (4.14) we know that all states $y_{u}$ are uniformly bounded. Moreover, we already have shown the $W(0, T)$-estimate for $y_{u}-\bar{y}$ in (4.15). In this way, we are able to bound the right hand sides of (4.19) against $\|u-\bar{u}\|_{L^{2}(Q)}$ and to verify the $W(0, T)$-estimate for $\varphi_{u}-\bar{\varphi}$ in (4.15).

For the $L^{\infty}$-estimate (4.16), we recall that the $L^{\infty}$-norm of the solution of a linear parabolic equation with bounded coefficients can be estimated against the 
$L^{p}$-norm of the right-hand side and to the $L^{\infty}$-norm of the initial data, provided that $p>N / 2+1$, see $[16$, Thm. 5.5]. (This result has to be applied to the equations for $\mathrm{e}^{-\mu t}\left(y_{u}-\bar{y}\right)$ and $\mathrm{e}^{-\mu t}\left(\varphi_{u}-\bar{\varphi}\right)$, respectively.)

In the terminal condition for $\varphi_{u}-\bar{\varphi}$ of $(4.19)$, we use that $\left\|y_{u}(T)-\bar{y}(T)\right\|_{L^{\infty}(\Omega)} \leq$ $\left\|y_{u}-\bar{y}\right\|_{L^{\infty}(Q)}$. The $L^{\infty}$ estimation of $\varphi_{u}-\bar{\varphi}$ in (4.16) is now straightforward.

Finally, we verify (4.17). To this aim, we set analogously $w(x, t)=\mathrm{e}^{-\mu t} \eta_{v}(x, t)$. Then, $w$ satisfies (4.18) with $\mathrm{e}^{-\mu t} v$ in the right hand side and $\bar{y}$ substituted for $\hat{y}_{u}$. Next, we fix $\mu$ large enough such that $\mathrm{e}^{-\mu t} \frac{\partial R}{\partial y}(x, t, \bar{y})+\mu \geq 0$ in $Q$. Multiplying the equation by $w$ and integrating in $Q$ we infer

$$
\frac{1}{2}\|w(T)\|_{L^{2}(\Omega)}^{2}+\|\nabla w\|_{L^{2}(Q)}^{2}+\|w\|_{L^{2}(Q)}^{2} \leq \int_{Q} \mathrm{e}^{-\mu t} v w d x d t \leq\|v\|_{L^{1}(Q)}\|w\|_{L^{\infty}(Q)} .
$$

From here we get

$$
\begin{aligned}
& \|w\|_{L^{2}(Q)}^{2}+\gamma\|w(T)\|_{L^{2}(\Omega)}^{2} \\
& \leq \max \{1, \gamma\}\left(\|w\|_{L^{2}(Q)}^{2}+\|w(T)\|_{L^{2}(\Omega)}^{2}\right) \leq C\|v\|_{L^{1}(Q)}\|v\|_{L^{p}(Q)} .
\end{aligned}
$$

Transforming back by $\eta_{v}(x, t)=\mathrm{e}^{\mu t} w(x, t)$, we obtain $(4.17)$.

Lemma 4 There exist a constant $C_{\eta}$ such that

$$
\left\|\eta_{u, v}-\eta_{v}\right\|_{W(0, T)} \leq C_{\eta}\left\|y_{u}-\bar{y}\right\|_{L^{\infty}(Q)}\left\|\eta_{v}\right\|_{L^{2}(Q)} \quad \forall v \in L^{2}(Q), \forall u \in \mathcal{U}_{\mathrm{ad}},
$$

where $\eta_{u, v}=G^{\prime}(u) v$ and $\eta_{v}=G^{\prime}(\bar{u}) v$. Moreover, a constant $C_{0}$ exists such that

$$
\left\|y_{u}-\bar{y}-\eta_{u-\bar{u}}\right\|_{W(0, T)} \leq C_{0}\left\|y_{u}-\bar{y}\right\|_{L^{\infty}(Q)}\left\|y_{u}-\bar{y}\right\|_{L^{2}(Q)} \quad \forall u \in \mathcal{U}_{\mathrm{ad}} .
$$

Proof Proceeding as in the last proof, we substitute $\eta_{u, v}-\eta_{v}=\mathrm{e}^{\mu t} w(x, t)$ with $\mu \geq-c_{R}$. Now, we subtract the equation satisfied by $\eta_{v}$ from that for $\eta_{u, v}$, perform the substitution and apply the mean value theorem to get

$$
\left\{\begin{array}{rlrl}
\frac{\partial w}{\partial t}-\Delta w+\left(\mathrm{e}^{-\mu t} \frac{\partial R}{\partial y}\left(x, t, y_{u}\right)+\mu\right) w & =-\mathrm{e}^{-\mu t} \frac{\partial^{2} R}{\partial y^{2}}\left(x, t, \tilde{y}_{u}\right)\left(y_{u}-\bar{y}\right) \eta_{v} & \text { in } Q \\
w & =0 & & \text { on } \Sigma \\
w(x, 0) & =0 & & \text { in } \Omega
\end{array}\right.
$$

where $\tilde{y}_{u}(x, t)=\bar{y}(x, t)+\vartheta(x, t)\left(y_{u}(x, t)-\bar{y}(x, t)\right), 0 \leq \vartheta(x, t) \leq 1$. Following the lines of the last proof, we obtain (4.20).

To show (4.21), we proceed similarly. We substitute $y_{u}-\bar{y}-\eta_{u-\bar{u}}=\mathrm{e}^{\mu t} \tilde{w}$ and perform the Taylor expansion

$$
R\left(x, t, y_{u}\right)=R(x, t, \bar{y})+\frac{\partial R}{\partial y}(x, t, \bar{y})\left(y_{u}-\bar{y}\right)+\frac{1}{2} \frac{\partial^{2} R}{\partial y^{2}}\left(x, t, \bar{y}+\theta\left(y_{u}-\bar{y}\right)\right)\left(y_{u}-\bar{y}\right)^{2}
$$

with $0 \leq \theta(x, t) \leq 1$. From the PDEs for $y_{u}, \bar{y}$, and $\eta_{u-\bar{u}}$, we deduce analogously to $(4.22)$

$$
\frac{\partial \tilde{w}}{\partial t}-\Delta \tilde{w}+\mathrm{e}^{-\mu t} \frac{\partial R}{\partial y}(x, t, \bar{y}) \tilde{w}+\mu \tilde{w}=-\mathrm{e}^{-\mu t} \frac{\partial^{2} R}{\partial y^{2}}\left(x, t, \bar{y}+\theta\left(y_{u}-\bar{y}\right)\right)\left(y_{u}-\bar{y}\right)^{2} \text { in } Q
$$

subject to the same homogeneous boundary and initial conditions as for $w$ above. Then the estimate (4.21) for $\tilde{w}=y_{u}-\bar{u}-\eta_{u-\bar{u}}$ follows as the one for $w$. 
For convenience of the reader we recall that, by the definition in Lemma 4, we have $\eta_{u, v}=G^{\prime}(u) v$ while $\eta_{v}=G^{\prime}(\bar{u}) v$ belongs to the fixed reference control $\bar{u}$.

Lemma 5 There exists a constant $M_{a, b}$ such that, for all $u \in \mathcal{U}_{\mathrm{ad}}$ and for all $v_{1}, v_{2} \in$ $L^{2}(Q)$, the following estimate holds

$$
\left|J^{\prime \prime}(u)\left(v_{1}, v_{2}\right)\right| \leq M_{a, b}\left(\left\|\eta_{v_{1}}\right\|_{L^{2}(Q)}\left\|\eta_{v_{2}}\right\|_{L^{2}(Q)}+\gamma\left\|\eta_{v_{1}}(T)\right\|_{L^{2}(\Omega)}\left\|\eta_{v_{2}}(T)\right\|_{L^{2}(\Omega)}\right) .
$$

Proof This estimate follows easily from the expression (2.6) of $J^{\prime \prime}$ and (4.14).

We also need the following preparatory result:

Lemma 6 For every $\rho>0$ there exists $\varepsilon>0$ such that

$$
\left|\left[J^{\prime \prime}(u)-J^{\prime \prime}(\bar{u})\right] v^{2}\right| \leq \rho\left(\left\|\eta_{v}\right\|_{L^{2}(Q)}^{2}+\gamma\left\|\eta_{v}(T)\right\|_{L^{2}(\Omega)}^{2}\right)
$$

holds for all $v \in L^{2}(Q)$ and for all $u \in \mathcal{U}_{\mathrm{ad}}$ such that $\left\|y_{u}-\bar{y}\right\|_{L^{\infty}(Q)}<\varepsilon$.

Proof From (2.6), it follows

$$
\begin{aligned}
{\left[J^{\prime \prime}(u)-J^{\prime \prime}(\bar{u})\right] v^{2} } & =\int_{Q}\left(\eta_{u, v}^{2}-\eta_{v}^{2}\right) d x d t \\
& +\int_{Q}\left\{\frac{\partial R}{\partial y}(x, t, \bar{y}) \bar{\varphi} \eta_{v}^{2}-\frac{\partial R}{\partial y}\left(x, t, y_{u}\right) \varphi_{u} \eta_{u, v}^{2}\right\} d x d t \\
& +\gamma \int_{\Omega}\left(\eta_{u, v}^{2}(T)-\eta_{v}^{2}(T)\right) d x=: I_{1}+I_{2}+I_{3}
\end{aligned}
$$

Now we discuss the estimation of $I_{1}, I_{2}$, and $I_{3}$. First, (4.20) yields that

$$
\begin{gathered}
\left\|\eta_{u, v}-\eta_{v}\right\|_{L^{2}(Q)} \leq\left\|\eta_{u, v}-\eta_{v}\right\|_{W(0, T)} \leq C_{\eta}\left\|y_{u}-\bar{y}\right\|_{L^{\infty}(Q)}\left\|\eta_{v}\right\|_{L^{2}(Q)} \\
\left\|\eta_{u, v}(T)-\eta_{v}(T)\right\|_{L^{2}(\Omega)} \leq C\left\|\eta_{u, v}-\eta_{v}\right\|_{W(0, T)} \\
\leq C C_{\eta}\left\|y_{u}-\bar{y}\right\|_{L^{\infty}(Q)}\left\|\eta_{v}\right\|_{L^{2}(Q)} .
\end{gathered}
$$

From here, we also get

$$
\begin{aligned}
& \left\|\eta_{u, v}\right\|_{L^{2}(Q)} \leq\left(1+C_{\eta}\left\|y_{u}-\bar{y}\right\|_{L^{\infty}(Q)}\right)\left\|\eta_{v}\right\|_{L^{2}(Q)} \\
& \left\|\eta_{u, v}(T)\right\|_{L^{2}(\Omega)} \leq\left\|\eta_{u, v}(T)-\eta_{v}(T)\right\|_{L^{2}(\Omega)}+\left\|\eta_{v}(T)\right\|_{L^{2}(\Omega)} \\
& \leq C C_{\eta}\left\|y_{u}-\bar{y}\right\|_{L^{\infty}(Q)}\left\|\eta_{v}\right\|_{L^{2}(Q)}+\left\|\eta_{v}(T)\right\|_{L^{2}(\Omega)} .
\end{aligned}
$$

By (4.25) and (4.27), we estimate $I_{1}$,

$$
\begin{aligned}
\left|I_{1}\right| & \leq\left\|\eta_{u, v}+\eta_{v}\right\|_{L^{2}(Q)}\left\|\eta_{u, v}-\eta_{v}\right\|_{L^{2}(Q)} \\
& \leq\left(2+C_{\eta}\left\|y_{u}-\bar{y}\right\|_{L^{\infty}(Q)}\right) C_{\eta}\left\|y_{u}-\bar{y}\right\|_{L^{\infty}(Q)}\left\|\eta_{v}\right\|_{L^{2}(Q)}^{2}
\end{aligned}
$$


$I_{3}$ is handled by (4.26) and (4.28) as follows

$$
\begin{aligned}
& \left|I_{3}\right| \leq \gamma\left\|\eta_{u, v}(T)+\eta_{v}(T)\right\|_{L^{2}(\Omega)}\left\|\eta_{u, v}(T)-\eta_{v}(T)\right\|_{L^{2}(\Omega)} \\
& \leq \gamma\left[C^{2} C_{\eta}^{2}\left\|y_{u}-\bar{y}\right\|_{L^{\infty}(Q)}^{2}\left\|\eta_{v}\right\|_{L^{2}(Q)}^{2}\right. \\
& \left.\quad \quad+C C_{\eta}\left\|y_{u}-\bar{y}\right\|_{L^{\infty}(Q)} 2\left\|\eta_{v}\right\|_{L^{2}(Q)}\left\|\eta_{v}(T)\right\|_{L^{2}(\Omega)}\right] \\
& \quad \leq \gamma\left\|y_{u}-\bar{y}\right\|_{L^{\infty}(Q)}\left\{C^{2} C_{\eta}^{2}\left(1+\left\|y_{u}-\bar{y}\right\|_{L^{\infty}(Q)}\right)\left\|\eta_{v}\right\|_{L^{2}(Q)}^{2}+\left\|\eta_{v}(T)\right\|_{L^{2}(\Omega)}^{2}\right\} .
\end{aligned}
$$

Finally, we deal with $I_{2}$ by

$$
\begin{aligned}
\left|I_{2}\right| \leq & \left\|\frac{\partial R}{\partial y}(x, t, \bar{y})-\frac{\partial R}{\partial y}\left(x, t, y_{u}\right)\right\|_{L^{\infty}(Q)}\|\bar{\varphi}\|_{L^{\infty}(Q)}\left\|\eta_{v}\right\|_{L^{2}(Q)}^{2} \\
& +\left\|\frac{\partial R}{\partial y}\left(x, t, y_{u}\right)\right\|_{L^{\infty}(Q)}\left\|\bar{\varphi}-\varphi_{u}\right\|_{L^{\infty}(Q)}\left\|\eta_{v}\right\|_{L^{2}(Q)}^{2} \\
& +\left\|\frac{\partial R}{\partial y}\left(x, t, y_{u}\right)\right\|_{L^{\infty}(Q)}\left\|\varphi_{u}\right\|_{L^{\infty}(Q)}\left\|\eta_{v}^{2}-\eta_{u, v}^{2}\right\|_{L^{1}(Q)} .
\end{aligned}
$$

The first term is handled with the assumption (1.6) and (4.14). Thanks to (4.16), the second term can be estimated by $C^{\prime}\left\|y_{u}-\bar{y}\right\|_{L^{\infty}(Q)}\left\|\eta_{v}\right\|_{L^{2}(Q)}^{2}$ with some constant $C^{\prime}$. The third term is handled as $I_{1}$. The statement of the lemma is a straightforward consequence of the obtained estimates.

Proof of Theorem 8. In view of Lemma 6, an $\varepsilon_{0}>0$ exists such that, for all $u \in \mathcal{U}_{\text {ad }}$ with $\left\|y_{u}-\bar{y}\right\|_{L^{\infty}(Q)}<\varepsilon_{0}$,

$$
\left|\left[J^{\prime \prime}(u)-J^{\prime \prime}(\bar{u})\right] v^{2}\right| \leq \frac{\sigma}{8}\left\{\left\|\eta_{v}\right\|_{L^{2}(Q)}^{2}+\gamma\left\|\eta_{v}(T)\right\|_{L^{2}(\Omega)}^{2}\right\} \forall v \in L^{2}(Q) .
$$

Invoking Lemma 3, we find that

$$
\left\|y_{u}-\bar{y}\right\|_{L^{\infty}(Q)} \leq \frac{C_{N}}{C_{\infty}}\|u-\bar{u}\|_{L^{2 N}(Q)} \leq \frac{C_{N}}{C_{\infty}}(b-a)^{1-1 / N}\|u-\bar{u}\|_{L^{2}(Q)}^{1 / N} .
$$

Now we select $\varepsilon_{1}$ such that

$$
0<\varepsilon_{1} \leq\left(\frac{C_{\infty} \varepsilon_{0}}{C_{N}(b-a)^{1-1 / N}}\right)^{N} .
$$

Then $\left\|y_{u}-\bar{y}\right\|_{L^{\infty}(Q)}<\varepsilon_{0}$ if $\|u-\bar{u}\|_{L^{2}(Q)}<\varepsilon_{1}$. Hence, (4.29) is true in $\mathcal{U}_{\mathrm{ad}} \cap B_{\varepsilon_{1}}(\bar{u})$,

$\left|\left[J^{\prime \prime}(u)-J^{\prime \prime}(\bar{u})\right] v^{2}\right| \leq \frac{\sigma}{8}\left\{\left\|\eta_{v}\right\|_{L^{2}(Q)}^{2}+\gamma\left\|\eta_{v}(T)\right\|_{L^{2}(\Omega)}^{2}\right\} \forall v \in L^{2}(Q), u \in \mathcal{U}_{\mathrm{ad}} \cap B_{\varepsilon_{1}}(\bar{u})$.

Now we show (4.13) with $\varepsilon=\min \left\{\varepsilon_{1}, \varepsilon_{2}\right\}$, where $\varepsilon_{2}$ is defined below. Select $u \in$ $\mathcal{U}_{\text {ad }} \cap B_{\varepsilon}(\bar{u})$ and denote by $Q_{v}$ the set of points $(x, t) \in Q$ such that (4.9) is not satisfied by $(u-\bar{u})(x, t)$. Moreover, set $v=(u-\bar{u}) \chi_{Q_{v}}$, where $\chi_{Q_{v}}$ is the characteristic function of $Q_{v}$, and $w=(u-\bar{u})-v$. By definition, $w(x, t)$ satisfies (4.9) for every $(x, t) \in Q$, and hence $w \in C_{\bar{u}}^{\tau}$. 
From (4.10) and (4.30), we infer

$$
\begin{aligned}
J(u)-J(\bar{u}) \geq J^{\prime}(\bar{u})(u-\bar{u})+\frac{1}{2} J^{\prime \prime}(\bar{u}+\theta(u-\bar{u}))(u-\bar{u})^{2} \\
\geq \tau\|v\|_{L^{1}\left(Q_{v}\right)}+\frac{1}{2} J^{\prime \prime}(\bar{u})(u-\bar{u})^{2} \\
\quad+\frac{1}{2}\left[J^{\prime \prime}(\bar{u}+\theta(u-\bar{u}))-J^{\prime \prime}(\bar{u})\right](u-\bar{u})^{2} \\
\geq \tau\|v\|_{L^{1}(Q)}+\frac{1}{2} J^{\prime \prime}(\bar{u}) w^{2}+\frac{1}{2} J^{\prime \prime}(\bar{u}) v^{2}+J^{\prime \prime}(\bar{u})(v, w) \\
-\frac{\sigma}{16}\left\{\left\|\eta_{u-\bar{u}}\right\|_{L^{2}(Q)}^{2}+\gamma\left\|\eta_{u-\bar{u}}(T)\right\|_{L^{2}(\Omega)}^{2}\right\} .
\end{aligned}
$$

Invoking (4.17), we get

$$
\begin{aligned}
\left\|\eta_{v}\right\|_{L^{2}(Q)}^{2}+\gamma\left\|\eta_{v}(T)\right\|_{L^{2}(\Omega)}^{2} & \leq C_{1}(b-a)^{1-1 / N}\|u-\bar{u}\|_{L^{2}(Q)}^{1 / N}\|v\|_{L^{1}(Q)} \\
& \leq C_{1}(b-a)^{1-1 / N} \varepsilon_{2}^{1 / N}\|v\|_{L^{1}(Q)} .
\end{aligned}
$$

From here it follows

$$
\|v\|_{L^{1}(Q)} \geq \frac{1}{C_{1}(b-a)^{1-1 / N} \varepsilon_{2}^{1 / N}}\left\{\left\|\eta_{v}\right\|_{L^{2}(Q)}^{2}+\gamma\left\|\eta_{v}(T)\right\|_{L^{2}(\Omega)}^{2}\right\}
$$

Inserting this inequality in (4.31) and using (4.12) and (4.23), we continue by

$$
\begin{aligned}
J(u)-J(\bar{u}) & \geq \frac{\tau}{C_{1}(b-a)^{1-1 / N} \varepsilon_{2}^{1 / N}}\left\{\left\|\eta_{v}\right\|_{L^{2}(Q)}^{2}+\left\|\eta_{v}(T)\right\|_{L^{2}(\Omega)}^{2}\right\} \\
& +\frac{\sigma}{2}\left\{\left\|\eta_{w}\right\|_{L^{2}(Q)}^{2}+\gamma\left\|\eta_{w}(T)\right\|_{L^{2}(\Omega)}^{2}\right\}-\frac{M_{a, b}}{2}\left\{\left\|\eta_{v}\right\|_{L^{2}(Q)}^{2}+\gamma\left\|\eta_{v}(T)\right\|_{L^{2}(\Omega)}^{2}\right\} \\
& -M_{a, b}\left\{\left\|\eta_{v}\right\|_{L^{2}(Q)}\left\|\eta_{w}\right\|_{L^{2}(Q)}+\gamma\left\|\eta_{v}(T)\right\|_{L^{2}(\Omega)}\left\|\eta_{w}(T)\right\|_{L^{2}(\Omega)}\right\} \\
& -\frac{\sigma}{16}\left\{\left\|\eta_{u-\bar{u}}\right\|_{L^{2}(Q)}^{2}+\gamma\left\|\eta_{u-\bar{u}}(T)\right\|_{L^{2}(\Omega)}^{2}\right\}
\end{aligned}
$$

and, with Young's inequality,

$$
\begin{aligned}
\geq & \left(\frac{\tau}{C_{1}(b-a)^{1-1 / N} \varepsilon_{2}^{1 / N}}-\frac{M_{a, b}}{2}-\frac{M_{a, b}^{2}}{\sigma}\right)\left\{\left\|\eta_{v}\right\|_{L^{2}(Q)}^{2}+\gamma\left\|\eta_{v}(T)\right\|_{L^{2}(\Omega)}^{2}\right\} \\
& +\frac{\sigma}{4}\left\{\left\|\eta_{w}\right\|_{L^{2}(Q)}^{2}+\gamma\left\|\eta_{w}(T)\right\|_{L^{2}(\Omega)}^{2}\right\}-\frac{\sigma}{16}\left\{\left\|\eta_{u-\bar{u}}\right\|_{L^{2}(Q)}^{2}+\gamma\left\|\eta_{u-\bar{u}}(T)\right\|_{L^{2}(\Omega)}^{2}\right\} .
\end{aligned}
$$

Now we fix $\varepsilon_{2}>0$ satisfying

$$
\frac{\tau}{C_{1}(b-a)^{1-1 / N} \varepsilon_{2}^{1 / N}}-\frac{M_{a, b}}{2}-\frac{M_{a, b}^{2}}{\sigma} \geq \frac{\sigma}{4}
$$


Inserting this inequality in (4.32) we obtain

$$
\begin{gathered}
J(u)-J(\bar{u}) \geq \frac{\sigma}{4}\left\{\left\|\eta_{v}\right\|_{L^{2}(Q)}^{2}+\gamma\left\|\eta_{v}(T)\right\|_{L^{2}(\Omega)}^{2}+\left\|\eta_{w}\right\|_{L^{2}(Q)}^{2}+\gamma\left\|\eta_{w}(T)\right\|_{L^{2}(\Omega)}^{2}\right\} \\
-\frac{\sigma}{16}\left\{\left\|\eta_{u-\bar{u}}\right\|_{L^{2}(Q)}^{2}+\gamma\left\|\eta_{u-\bar{u}}(T)\right\|_{L^{2}(\Omega)}^{2}\right\} \\
\geq \frac{\sigma}{8}\left\{\left\|\eta_{v+w}\right\|_{L^{2}(Q)}^{2}+\gamma\left\|\eta_{v+w}(T)\right\|_{L^{2}(\Omega)}^{2}\right\} \\
\quad-\frac{\sigma}{16}\left\{\left\|\eta_{u-\bar{u}}\right\|_{L^{2}(Q)}^{2}+\gamma\left\|\eta_{u-\bar{u}}(T)\right\|_{L^{2}(\Omega)}^{2}\right\} \\
=\frac{\sigma}{16}\left\{\left\|\eta_{u-\bar{u}}\right\|_{L^{2}(Q)}^{2}+\gamma\left\|\eta_{u-\bar{u}}(T)\right\|_{L^{2}(\Omega)}^{2}\right\}
\end{gathered}
$$

(notice that $v+w=u-\bar{u}$ ). Hence, (4.13) holds with $\varepsilon=\min \left\{\varepsilon_{1}, \varepsilon_{2}\right\}$.

Corollary 3 ( $\nu=0$; Sufficient condition for a weak local solution) Under the assumptions of Theorem 8 , there exist $\varepsilon>0$ and $\delta>0$ such that

$$
J(\bar{u})+\frac{\delta}{2}\left\{\left\|y_{u}-\bar{y}\right\|_{L^{2}(Q)}^{2}+\gamma\left\|y_{u}(T)-\bar{y}(T)\right\|_{L^{2}(\Omega)}^{2}\right\} \leq J(u) \quad \forall u \in \mathcal{U}_{\mathrm{ad}} \cap B_{\varepsilon}(\bar{u}) .
$$

Proof We define $w=y_{u}-\bar{y}-\eta_{u-\bar{u}}$. Then the estimate (4.21) yields that

$$
\|w\|_{W(0, T)} \leq C_{0}\left\|y_{u}-\bar{y}\right\|_{L^{\infty}(Q)}\left\|y_{u}-\bar{y}\right\|_{L^{2}(Q)} \quad \forall u \in \mathcal{U}_{\mathrm{ad}} .
$$

Let now $\varepsilon$ be as in Theorem 8 and take $\varepsilon_{0} \leq \varepsilon$ such that $C_{0} \varepsilon_{0}<\frac{1}{2}$. Then, for $\left\|y_{u}-\bar{y}\right\|_{L^{\infty}(Q)}<\varepsilon_{0}$ we have

$$
\left\|y_{u}-\bar{y}\right\|_{L^{2}(Q)} \leq\|w\|_{L^{2}(Q)}+\left\|\eta_{u-\bar{u}}\right\|_{L^{2}(Q)} \leq \frac{1}{2}\left\|y_{u}-\bar{y}\right\|_{L^{2}(Q)}+\left\|\eta_{u-\bar{u}}\right\|_{L^{2}(Q)},
$$

hence, moving the term $\frac{1}{2}\left\|y_{u}-\bar{y}\right\|_{L^{2}(Q)}$ to the other side,

$$
\left\|y_{u}-\bar{y}\right\|_{L^{2}(Q)} \leq 2\left\|\eta_{u-\bar{u}}\right\|_{L^{2}(Q)} \quad \forall u \in \mathcal{U}_{\mathrm{ad}}:\left\|y_{u}-\bar{y}\right\|_{L^{\infty}(Q)}<\varepsilon_{0} .
$$

Moreover, we have

$$
\begin{aligned}
\| y_{u}(T) & -\bar{y}(T)\left\|_{L^{2}(\Omega)} \leq\right\| w(T)\left\|_{L^{2}(\Omega)}+\right\| \eta_{u-\bar{u}}(T) \|_{L^{2}(\Omega)} \\
& \leq C_{2}\|w\|_{W(0, T)}+\left\|\eta_{u-\bar{u}}(T)\right\|_{L^{2}(\Omega)} \\
& \leq \frac{C_{2}}{2}\left\|y_{u}-\bar{y}\right\|_{L^{2}(Q)}+\left\|\eta_{u-\bar{u}}(T)\right\|_{L^{2}(\Omega)} \leq C_{2}\left\|\eta_{u-\bar{u}}\right\|_{L^{2}(Q)}+\left\|\eta_{u-\bar{u}}(T)\right\|_{L^{2}(\Omega)} .
\end{aligned}
$$

Therefore, we get from the last two estimates

$$
\left\|y_{u}-\bar{y}\right\|_{L^{2}(Q)}^{2}+\gamma\left\|y_{u}(T)-\bar{y}(T)\right\|_{L^{2}(\Omega)}^{2} \leq C_{3}\left\{\left\|\eta_{u-\bar{u}}\right\|_{L^{2}(Q)}^{2}+\gamma\left\|\eta_{u-\bar{u}}(T)\right\|_{L^{2}(\Omega)}^{2}\right\}
$$

$\forall u \in \mathcal{U}_{\text {ad }}$ such that $\left\|y_{u}-\bar{y}\right\|_{L^{\infty}(Q)}<\varepsilon_{0}$. Finally, we take $0<\delta \leq \frac{\sigma}{8 C_{3}}$ and $0<\varepsilon \leq \varepsilon_{0}$ so that $\left\|y_{u}-\bar{y}\right\|_{L^{\infty}(Q)}<\varepsilon_{0}$ for every $u \in \mathcal{U}_{\mathrm{ad}} \cap B_{\varepsilon}(\bar{u})$. Then, (4.33) follows from (4.13). 
The growth condition (4.33) is valid in a ball around $\bar{u}$, hence we obtained a result on local optimality in weak sense. We were not able to prove that, under these assumptions based on the cone $C_{\bar{u}}^{\tau}$, the solution is locally optimal in strong sense. To deal with this problem, we introduce another extended cone by

$$
\begin{aligned}
& E_{\bar{u}}^{\tau}=\left\{v \in L^{2}(Q) \text { satisfying }(4.1)\right. \text { and } \\
& \left.\qquad J^{\prime}(\bar{u}) v \leq \tau\left(\left\|\eta_{v}\right\|_{L^{2}(Q)}+\gamma\left\|\eta_{v}(T)\right\|_{L^{2}(\Omega)}\right)\right\}
\end{aligned}
$$

From Lemma 1,(i) we infer that $C_{\bar{u}} \subset E_{\bar{u}}^{\tau}$ for every $\tau>0$. Thus the cone $E_{\bar{u}}^{\tau}$ is a small extension of $C_{\bar{u}}$. We are able to prove the following result on second order sufficiency that is based on $E_{\bar{u}}^{\tau}$ :

Theorem 9 Let $\bar{u} \in \mathcal{U}_{\mathrm{ad}}$, along with the adjoint state $\bar{\varphi}$, satisfy the variational inequality (3.2). Assume also that $\tau>0$ and $\sigma>0$ exist such that

$$
J^{\prime \prime}(\bar{u}) v^{2} \geq \sigma\left(\left\|\eta_{v}\right\|_{L^{2}(Q)}^{2}+\gamma\left\|\eta_{v}(T)\right\|_{L^{2}(\Omega)}^{2}\right) \quad \forall v \in E_{\bar{u}}^{\tau}
$$

Then, there exists $\varepsilon>0$ such that

$J\left(\bar{u}_{\nu}\right)+\frac{\delta}{2}\left\{\left\|\eta_{u-\bar{u}}\right\|_{L^{2}(Q)}^{2}+\gamma\left\|\eta_{u-\bar{u}}(T)\right\|_{L^{2}(\Omega)}^{2}\right\} \leq J(u) \forall u \in \mathcal{U}_{\mathrm{ad}}:\left\|y_{u}-\bar{y}\right\|_{L^{\infty}(Q)}<\varepsilon$

Proof Proceeding completely analogous to [10], we obtain a constant $M>0$ such that

$$
\left\|\eta_{u-\bar{u}}\right\|_{L^{2}(Q)}+\gamma\left\|\eta_{u-\bar{u}}(T)\right\|_{L^{2}(\Omega)} \leq M\left\|y_{u}-\bar{y}\right\|_{L^{\infty}(Q)} \quad \forall u \in \mathcal{U}_{\mathrm{ad}}
$$

Define

$$
\varepsilon_{1}=\frac{4 \tau C_{\gamma}}{M\left(\sigma+2 M_{a, b}\right)} \quad \text { with } \quad C_{\gamma}=\left\{\begin{array}{l}
1 \text { if } \gamma=0 \text { or } \gamma \geq 1 \\
\gamma \text { if } 0<\gamma<1
\end{array}\right.
$$

where $M_{a, b}$ was introduced in Lemma 5 . From Lemma 6, we deduce the existence of $\varepsilon_{2}>0$ such that $\forall u \in \mathcal{U}_{\text {ad }}$ with $\left\|y_{u}-\bar{y}\right\|_{L^{\infty}(Q)}<\varepsilon_{2}$

$$
\left|\left[J^{\prime \prime}(u)-J^{\prime \prime}(\bar{u})\right] v^{2}\right| \leq \frac{\sigma}{2}\left\{\left\|\eta_{v}\right\|_{L^{2}(Q)}^{2}+\gamma\left\|\eta_{v}(T)\right\|_{L^{2}(\Omega)}^{2}\right\} \forall v \in L^{2}(Q)
$$

With these prerequisites, we are able to verify (4.35) with $\varepsilon=\min \left\{\varepsilon_{1}, \varepsilon_{2}\right\}$. To this aim, we select $u \in \mathcal{U}_{\text {ad }}$ such that $\left\|y_{u}-\bar{y}\right\|_{L^{\infty}(Q)}<\varepsilon$ and distinguish between two cases.

CASE I: $u-\bar{u} \notin E_{\bar{u}}^{\tau}$. This is the case, where $J^{\prime}(\bar{u})(u-\bar{u})$ is sufficiently big to ensure local optimality without the coercivity assumption (4.34). 
Here we estimate as follows:

$$
\begin{aligned}
& J(u)-J(\bar{u}) \geq J^{\prime}(\bar{u})(u-\bar{u})+\frac{1}{2} J^{\prime \prime}(\bar{u}+\theta(u-\bar{u}))(u-\bar{u})^{2} \\
&> \tau\left\{\left\|\eta_{u-\bar{u}}\right\|_{L^{2}(Q)}+\gamma\left\|\eta_{u-\bar{u}}(T)\right\|_{L^{2}(\Omega)}\right\} \\
& \quad-\frac{M_{a, b}}{2}\left\{\left\|\eta_{u-\bar{u}}\right\|_{L^{2}(Q)}^{2}+\gamma\left\|\eta_{u-\bar{u}}(T)\right\|_{L^{2}(\Omega)}^{2}\right\} \\
& \geq \frac{\tau}{M \varepsilon}\left\{\left\|\eta_{u-\bar{u}}\right\|_{L^{2}(Q)}+\gamma\left\|\eta_{u-\bar{u}}(T)\right\|_{L^{2}(\Omega)}\right\}^{2} \\
& \quad-\frac{M_{a, b}}{2}\left\{\left\|\eta_{u-\bar{u}}\right\|_{L^{2}(Q)}^{2}+\gamma\left\|\eta_{u-\bar{u}}(T)\right\|_{L^{2}(\Omega)}^{2}\right\} \\
& \geq\left(\frac{\tau C_{\gamma}}{M \varepsilon}-\frac{M_{a, b}}{2}\right)\left\{\left\|\eta_{u-\bar{u}}\right\|_{L^{2}(Q)}^{2}+\gamma\left\|\eta_{u-\bar{u}}(T)\right\|_{L^{2}(\Omega)}^{2}\right\} \\
& \geq \frac{\sigma}{4}\left\{\left\|\eta_{u-\bar{u}}\right\|_{L^{2}(Q)}^{2}+\gamma\left\|\eta_{u-\bar{u}}(T)\right\|_{L^{2}(\Omega)}^{2}\right\} .
\end{aligned}
$$

Notice that (4.36) yields $(M \varepsilon)^{-1}\left(\left\|\eta_{u-\bar{u}}\right\|_{L^{2}(Q)}+\gamma\left\|\eta_{u-\bar{u}}(T)\right\|_{L^{2}(\Omega)}\right) \leq 1$.

CASE II: $u-\bar{u} \in E_{\bar{u}}^{\tau}$. Here, the term $J^{\prime}(\bar{u})(u-\bar{u})$ is so small that the coercivity condition (4.34) has to be invoked.

This time, we use (3.2), (4.34) and (4.37) to get

$$
\begin{aligned}
J(u)-J(\bar{u}) & =J^{\prime}(\bar{u})(u-\bar{u})+\frac{1}{2} J^{\prime \prime}(\bar{u}+\theta(u-\bar{u}))(u-\bar{u})^{2} \\
& =\int_{Q} \bar{\varphi}(u-\bar{u}) d x d t+\frac{1}{2} J^{\prime \prime}(\bar{u}+\theta(u-\bar{u}))(u-\bar{u})^{2} \\
& \geq \frac{1}{2} J^{\prime \prime}(\bar{u})(u-\bar{u})^{2}+\frac{1}{2}\left[J^{\prime \prime}(\bar{u}+\theta(u-\bar{u}))-J^{\prime \prime}(\bar{u})\right](u-\bar{u})^{2} \\
& \geq \frac{\sigma}{2}\left\{\left\|\eta_{u-\bar{u}}\right\|_{L^{2}(Q)}^{2}+\gamma\left\|\eta_{u-\bar{u}}(T)\right\|_{L^{2}(\Omega)}^{2}\right\} \\
& \quad-\frac{\sigma}{4}\left\{\left\|\eta_{u-\bar{u}}\right\|_{L^{2}(Q)}^{2}+\gamma\left\|\eta_{u-\bar{u}}(T)\right\|_{L^{2}(\Omega)}^{2}\right\} \\
& =\frac{\sigma}{4}\left\{\left\|\eta_{u-\bar{u}}\right\|_{L^{2}(Q)}^{2}+\gamma\left\|\eta_{u-\bar{u}}(T)\right\|_{L^{2}(\Omega)}^{2}\right\} .
\end{aligned}
$$

Corollary 4 ( $\nu=0$; Sufficient condition for a strong local minimum) If $\bar{u}$ satisfies the assumptions of Theorem 9, then there exist $\delta>0$ and $\varepsilon>0$ such that

$$
J(\bar{u})+\frac{\delta}{2}\left\{\left\|y_{u}-\bar{y}\right\|_{L^{2}(Q)}^{2}+\left\|y_{u}(T)-\bar{y}(T)\right\|_{L^{2}(\Omega)}^{2}\right\} \leq J(u)
$$

holds for all $u \in \mathcal{U}_{\text {ad }}$ with $\left\|y_{u}-\bar{y}\right\|_{L^{\infty}(Q)}<\varepsilon$.

The proof of this corollary is completely analogous to that of Corollary 3. We take $\varepsilon_{0}$ and $C_{3}$ as in the proof of Corollary 3, and $\delta$ and $\varepsilon$ as in the statement of Theorem 9. Then the inequality (4.38) follows by substituting $\varepsilon$ for $\min \left\{\varepsilon_{0}, \varepsilon\right\}$ and $\delta$ for $\delta / C_{3}$. 


\section{Applications to the Stability Analysis with Respect to Perturbations}

In this section we explain how our second order sufficient conditions for strong local solutions can be applied to proving stability estimates for the local solution with respect to certain perturbations in the data of our optimal control problem. Here, we concentrate on the case $\nu=0$, where the second-order sufficient optimality conditions known from earlier papers cannot be applied.

First, we consider perturbations of the desired state $y_{Q}$ and second the behavior of the local solution for the Tikhonov regularization parameter $\nu$ tending to zero. The second problem is also an issue of stability analysis, because a small Tikhonov parameter can be viewed as a perturbation of the reference parameter $\nu=0$.

\subsection{Perturbation of $y_{Q}$ and $y_{T}$}

Assume that, for all $\varepsilon>0$ a perturbed desired state $y_{Q}^{\varepsilon} \in L^{p}(Q), p>N / 2+1$, and a perturbed desired final state $y_{T}^{\varepsilon} \in L^{\infty}(\Omega)$ are given such that

$$
\left\|y_{Q}^{\varepsilon}-y_{Q}\right\|_{L^{2}(Q)}+\gamma\left\|y_{T}^{\varepsilon}-y_{T}\right\|_{L^{2}(\Omega)} \leq C \varepsilon \quad \forall \varepsilon>0
$$

holds with some constant $C>0$.

Associated with these perturbed target functions, we define the family of perturbed objective functionals

$$
J^{\varepsilon}(u)=\frac{1}{2} \int_{Q}\left(y_{u}-y_{Q}^{\varepsilon}\right)^{2} d x d t+\frac{\gamma}{2} \int_{\Omega}\left(y_{u}(T)-y_{T}^{\varepsilon}\right)^{2} d x
$$

and consider the family of perturbed optimal control problems

$$
\left(\mathrm{P}^{\varepsilon}\right)\left\{\operatorname{Min}_{u \in \mathcal{U}_{\mathrm{ad}}} J^{\varepsilon}(u) .\right.
$$

Notice that the perturbed functionals do not include a Tikhonov regularization term, i.e. we have $\nu=0$.

It follows by the same arguments as for the unperturbed problem that for each $\varepsilon>0$ at least one optimal control $\bar{u}^{\varepsilon}$ exists. We denote by $\bar{y}^{\varepsilon}$ the associated optimal state and investigate the behavior $\bar{y}^{\varepsilon}$ for $\varepsilon \searrow 0$.

First, we quote a result on convergence from [9] that does not yet contain information on the rate of convergence. Notice that the set $\mathcal{U}_{\text {ad }}$ is bounded. Therefore, a subsequence of $\bar{u}^{\varepsilon}$ can be selected that converges weakly in $L^{2}(Q)$. By selecting a subsequence if necessary, we can assume weak convergence of $\left\{\bar{u}^{\varepsilon}\right\}_{\varepsilon}$.

Theorem 10 ( $\nu=0$; Convergence for $\varepsilon \searrow 0)$ If $\left\{\bar{u}^{\varepsilon}\right\}_{\varepsilon}$ is any sequence of optimal controls of the problems $\left(\mathrm{P}^{\varepsilon}\right)$ that converges weakly in $L^{2}(Q)$ to some $\bar{u}$, then $\bar{u}$ is optimal for $(\mathrm{P})$ and

$$
\lim _{\varepsilon \searrow 0}\left\|\bar{y}^{\varepsilon}-\bar{y}\right\|_{L^{\infty}(Q)}=0 .
$$

Conversely, let $\bar{u}$ be a strict locally optimal control of $(\mathrm{P})$. Then there exists a sequence $\left\{\bar{u}^{\varepsilon}\right\}_{\varepsilon}$ of locally optimal controls of $\left(\mathrm{P}^{\varepsilon}\right)$ converging weakly to $\bar{u}$. For this sequence, (5.3) holds as well. Furthermore, a radius $\rho>0$ exists such that every $\bar{u}^{\varepsilon}$ affords a global minimum to $J^{\varepsilon}$ with respect to all elements $u \in \mathcal{U}_{\text {ad }}$ such that $\left\|y_{u}-\bar{y}\right\|_{\infty} \leq \rho$. 
We refer to the proof in [10]. This theorem ensures the convergence of $\bar{y}^{\varepsilon}$ to $\bar{y}$ as $\varepsilon \searrow 0$. The associated rate of convergence is the subject of the next result.

Theorem 11 ( $\nu=0$; Lipschitz stability for $\varepsilon \searrow 0$ ) Assume that $\bar{u}$ is a locally optimal control of $(\mathrm{P})$ that satisfies the second order sufficient optimality condition (4.34). Let $\left\{\bar{u}_{\varepsilon}\right\}$ be a sequence of locally optimal controls of $\left(\mathrm{P}^{\varepsilon}\right)$ that converges weakly to $\bar{u}$ in $L^{p}(Q)$ as $\varepsilon \searrow 0$ and has the properties stated in Theorem 10; denote the associated states by $\bar{y}$ and $\bar{y}_{\varepsilon}$, respectively. Then a number $C>0$ exists such that

$$
\left\|\bar{y}_{\varepsilon}-\bar{y}\right\|_{L^{2}(Q)}+\gamma\left\|\bar{y}_{\varepsilon}(T)-\bar{y}(T)\right\|_{L^{2}(\Omega)} \leq C \varepsilon
$$

Proof In view of the assumed properties of the sequence $\left\{\bar{u}^{\varepsilon}\right\}$ we have $\| \bar{y}_{\varepsilon}-$ $\bar{y} \|_{L^{\infty}(Q)} \rightarrow 0$ as $\varepsilon \searrow 0$. Consequently, for all sufficiently small $\varepsilon>0, \bar{y}_{\varepsilon}$ is so close to $\bar{y}$ that the quadratic growth condition (4.38) is satisfied by Corollary 4 . Invoking this growth condition, we proceed as follows:

$$
\begin{aligned}
J^{\varepsilon}(\bar{u}) & \geq J^{\varepsilon}\left(\bar{u}_{\varepsilon}\right)=J\left(\bar{u}_{\varepsilon}\right)+J^{\varepsilon}\left(\bar{u}_{\varepsilon}\right)-J\left(\bar{u}_{\varepsilon}\right) \\
& \geq J(\bar{u})+\frac{\delta}{2}\left(\left\|\bar{y}_{\varepsilon}-\bar{y}\right\|_{L^{2}(Q)}^{2}+\gamma\left\|\bar{y}_{\varepsilon}(T)-\bar{y}(T)\right\|_{L^{2}(\Omega)}^{2}\right)+J^{\varepsilon}\left(\bar{u}_{\varepsilon}\right)-J\left(\bar{u}_{\varepsilon}\right) .
\end{aligned}
$$

By shifting the the first and the last two terms to the left-hand side, this inequality becomes

$$
J^{\varepsilon}(\bar{u})-J(\bar{u})-\left(J^{\varepsilon}\left(\bar{u}_{\varepsilon}\right)-J\left(\bar{u}_{\varepsilon}\right)\right) \geq \frac{\delta}{2}\left(\left\|\bar{y}_{\varepsilon}-\bar{y}\right\|_{L^{2}(Q)}^{2}+\gamma\left\|\bar{y}_{\varepsilon}(T)-\bar{y}(T)\right\|_{L^{2}(\Omega)}^{2}\right) .
$$

The left side is an integral of various differences of squares. Expanding the squares, it can be considerably simplified. For instance, the tracking terms on $Q$ are handled as follows:

$$
\begin{aligned}
& \frac{1}{2} \int_{Q}\left(\left(\bar{y}-y_{Q}^{\varepsilon}\right)^{2}-\left(\bar{y}-y_{Q}\right)^{2}\right) d x d t-\frac{1}{2} \int_{Q}\left(\left(\bar{y}_{\varepsilon}-y_{Q}^{\varepsilon}\right)^{2}-\left(\bar{y}_{\varepsilon}-y_{Q}\right)^{2}\right) d x d t \\
& =\frac{1}{2} \int_{Q} 2\left(\bar{y}-\bar{y}_{\varepsilon}\right)\left(y_{Q}-y_{Q}^{\varepsilon}\right) d x d t \leq C \varepsilon\left\|\bar{y}-\bar{y}_{\varepsilon}\right\|_{L^{2}(Q)}
\end{aligned}
$$

in view of (5.1). In the same way, the integrals on $\Omega$ are simplified to finally obtain

$$
J^{\varepsilon}(\bar{u})-J(\bar{u})-\left(J^{\varepsilon}\left(\bar{u}_{\varepsilon}\right)-J\left(\bar{u}_{\varepsilon}\right)\right) \leq C \varepsilon\left(\left\|\bar{y}-\bar{y}_{\varepsilon}\right\|_{L^{2}(Q)}+\gamma\left\|\bar{y}(T)-\bar{y}_{\varepsilon}(T)\right\|_{L^{2}(\Omega)}\right) .
$$

Now we invoke (5.5) to conclude

$$
\begin{aligned}
C \varepsilon & \left(\left\|\bar{y}-\bar{y}_{\varepsilon}\right\|_{L^{2}(Q)}+\gamma\left\|\bar{y}(T)-\bar{y}_{\varepsilon}(T)\right\|_{L^{2}(\Omega)}\right) \\
& \geq \frac{\delta}{2}\left(\left\|\bar{y}_{\varepsilon}-\bar{y}\right\|_{L^{2}(Q)}^{2}+\gamma\left\|\bar{y}_{\varepsilon}(T)-\bar{y}(T)\right\|_{L^{2}(\Omega)}^{2}\right) \\
& \geq c\left(\left\|\bar{y}_{\varepsilon}-\bar{y}\right\|_{L^{2}(Q)}+\gamma\left\|\bar{y}_{\varepsilon}(T)-\bar{y}(T)\right\|_{L^{2}(\Omega)}\right)^{2} .
\end{aligned}
$$

The desired result follows immediately. 
Remark 3 Assume that the locally optimal control $\bar{u}$ considered in Theorem 10 is bang-bang. Then the second order sufficient optimality condition (4.34) - as one of the assumptions of Theorem 11 - can be replaced by the condition (4.12) and the result of the theorem remains true.

Indeed, if $\bar{u}$ is bang-bang and $\bar{u}_{\varepsilon} \rightarrow \bar{u}$ in $L^{2}(Q)$, then $\bar{u}_{\varepsilon} \rightarrow \bar{u}$ strongly in every space $L^{p}(Q)$ for $p<+\infty$; see [7, Theorem 4.4]. Therefore, $\bar{u}_{\varepsilon}$ belongs to any $L^{p}$ neighborhood $B_{r}(\bar{u})$ provided that $\varepsilon$ is sufficiently small. Then the assumptions of Corollary 3 are satisfied so that the quadratic growth condition (4.33) applies (substitute $B_{r}(\bar{u})$ for $B_{\varepsilon}(\bar{u})$ there). In view of that, the estimate (5.5) in the proof above holds for sufficiently small $\varepsilon>0$.

\subsection{Tikhonov parameter tending to zero}

As a further application of second order optimality conditions for strong local minima, we investigate the behavior of a sequence of optimal controls $\left\{\bar{u}_{\nu}\right\}_{\nu>0}$ of $\left(\mathrm{P}_{\nu}\right)$ and the corresponding states $\left\{\bar{y}_{\nu}\right\}_{\nu>0}$ as $\nu \searrow 0$.

Again, by the boundedness of $\mathcal{U}_{\mathrm{ad}}$, any sequence of solutions of $\left(\mathrm{P}_{\nu}\right)$ contains subsequences converging weakly in $L^{2}(Q)$.

Theorem 12 Let $\left\{\bar{u}_{\nu}\right\}_{\nu>0}$ be a sequence of global solutions of $\left(\mathrm{P}_{\nu}\right)$ such that $\bar{u}_{\nu} \rightarrow \bar{u}$ in $L^{2}(Q)$ for $\nu \searrow 0$. Then $\bar{u}$ is a global solution of $(\mathrm{P})$ and $\left\|\bar{u}_{\nu}-\bar{u}\right\|_{L^{2}(Q)} \rightarrow 0$. Moreover,

$$
\|\bar{u}\|_{L^{2}(Q)}=\min \left\{\|u\|_{L^{2}(Q)}: u \text { is a global solution of }(\mathrm{P})\right\} \text {. }
$$

Proof The boundedness of $\left\{\bar{u}_{\nu}\right\}_{\nu>0}$ in $L^{\infty}(Q)$ and the weak convergence $\bar{u}_{\nu} \rightarrow \bar{u}$ for $\nu \searrow 0$ in $L^{2}(Q)$ imply that $\bar{u}_{\nu} \rightarrow \bar{u}$ for $\nu \searrow 0$ in $L^{p}(Q)$ for any $1 \leq p<\infty$. Moreover, we have $\bar{u} \in \mathcal{U}_{\text {ad }}$. Let $\bar{y}_{\nu}=G\left(\bar{u}_{\nu}\right)$ and $\bar{y}=G(\bar{u})$.

Now we pass to the limit in the state equation (1.2) that is satisfied by $\bar{y}_{\nu}$. In view of (4.14) and (4.16), it is easy to confirm that $\bar{y}_{\nu} \rightarrow \bar{y}$ in $W(0, T)$. The embedding $W(0, T) \hookrightarrow L^{2}(Q)$ is known to be continuous; also the linear mapping $W(0, T) \ni w \mapsto w(T) \in L^{2}(\Omega)$ is continuous. The cost functional is convex with respect to $(y, u)$. Since $\bar{u}_{\nu}$ is a solution of $\left(\mathrm{P}_{\nu}\right)$, it follows

$$
J(\bar{u}) \leq \liminf _{\nu \searrow 0} J\left(\bar{u}_{\nu}\right) \leq \liminf _{\nu \searrow 0} J_{\nu}\left(\bar{u}_{\nu}\right) \leq \lim _{\nu \searrow 0} J_{\nu}(u)=J(u) \quad \forall u \in \mathcal{U}_{\mathrm{ad}} .
$$

Therefore, $\bar{u}$ is a solution of $(\mathrm{P})$.

Next, we verify the strong convergence of $\left\{\bar{u}_{\nu}\right\}_{\nu>0}$ to $\bar{u}$. Since $\bar{u}_{\nu}$ and $\bar{u}$ are solutions of $\left(\mathrm{P}_{\nu}\right)$ and $(\mathrm{P})$, respectively, we obtain

$$
J\left(\bar{u}_{\nu}\right)+\frac{\nu}{2}\left\|\bar{u}_{\nu}\right\|_{L^{2}(Q)}^{2}=J_{\nu}\left(\bar{u}_{\nu}\right) \leq J_{\nu}(\bar{u})=J(\bar{u})+\frac{\nu}{2}\|\bar{u}\|_{L^{2}(Q)}^{2} \leq J\left(\bar{u}_{\nu}\right)+\frac{\nu}{2}\|\bar{u}\|_{L^{2}(Q)}^{2} .
$$

Consequently, $\left\|\bar{u}_{\nu}\right\|_{L^{2}(Q)}^{2} \leq\|\bar{u}\|_{L^{2}(Q)}^{2}$ holds for every $\nu>0$. From here, we infer

$$
\|\bar{u}\|_{L^{2}(Q)} \leq \liminf _{\nu \searrow 0}\left\|\bar{u}_{\nu}\right\|_{L^{2}(Q)} \leq \limsup _{\nu \searrow 0}\left\|\bar{u}_{\nu}\right\|_{L^{2}(Q)} \leq\|\bar{u}\|_{L^{2}(Q)}
$$


and hence $\left\|\bar{u}_{\nu}\right\|_{L^{2}(Q)} \rightarrow\|\bar{u}\|_{L^{2}(Q)}$. This fact implies, along with the weak convergence $\bar{u}_{\nu} \rightarrow \bar{u}$, the strong convergence $\bar{u}_{\nu} \searrow \bar{u}$ in $L^{2}(Q)$. Finally, arguing as above, we obtain for any (global) solution $u$ of $(\mathrm{P})$ the inequality $\left\|\bar{u}_{\nu}\right\|_{L^{2}(Q)} \leq\|u\|_{L^{2}(Q)}$ $\forall \nu>0$. The minimum norm property (5.6) follows from

$$
\|\bar{u}\|_{L^{2}(Q)}=\lim _{\nu \searrow 0}\left\|\bar{u}_{\nu}\right\|_{L^{2}(Q)} \leq\|u\|_{L^{2}(Q)}
$$

Now, we formulate a converse result, namely that strict local solutions to $(\mathrm{P})$ can be approximated by local solutions of $\left(\mathrm{P}_{\nu}\right)$. This result is analogous to the second part of Theorem 10.

Theorem 13 Let $\bar{u}$ be a strict local solution of $(\mathrm{P})$. Then there exist constants $\rho>0$, $\bar{\nu}>0$, and a sequence $\left\{\bar{u}_{\nu}\right\}_{0<\nu<\bar{\nu}}$ of local solutions of $\left(\mathrm{P}_{\nu}\right)$ such that $\bar{u}_{\nu} \rightarrow \bar{u}$ in $L^{2}(Q)$ and every $\bar{u}_{\nu}$ affords a global minimum to $J_{\nu}$ in $\mathbb{K}_{\rho}=\mathcal{U}_{\mathrm{ad}} \cap \bar{B}_{\rho}(\bar{u})$.

Again, this is a fairly standard result. We refer the reader to [10].

We do not know the rate of convergence of global (local) solutions $\bar{u}_{\nu}$ of $\left(\mathrm{P}_{\nu}\right)$ to global (local) solutions $\bar{u}$ of $(\mathrm{P})$. Notice that the Tikhonov parameter $\nu$ vanishes in the limit, hence it should be difficult to find such a rate.

Instead, we are able to estimate the rate of convergence for the associated state functions, if certain second order sufficient optimality conditions are satisfied at $\bar{u}$.

The associated theorem below is applicable, if the coercivity condition is fulfilled in the form (4.12) (based on $C_{\bar{u}}^{\tau}$ ) or in the form (4.34) (based on $E_{\bar{u}}^{\tau}$ ). Notice that $y_{u} \neq y_{v}$ implies $u \neq v$. Therefore, the strong quadratic growth condition (4.38) ensures in particular that $\bar{u}$ is a strict local solution.

Theorem 14 (Hölder rate of convergence as $\nu \searrow 0$ ) Let $\bar{u}$ and $\left\{\bar{u}_{\nu}\right\}_{0<\nu \leq \bar{\nu}}$ be a sequence of local solutions as in Theorem 13. Assume that one of the coercivity conditions (4.12) or (4.34) is satisfied. Then, the equation

$$
\lim _{\nu \searrow 0} \frac{1}{\sqrt{\nu}}\left\{\left\|\bar{y}_{\nu}-\bar{y}\right\|_{L^{2}(Q)}+\gamma\left\|\bar{y}_{\nu}(T)-\bar{y}(T)\right\|_{L^{2}(\Omega)}\right\}=0
$$

is fulfilled, where $\bar{y}_{\nu}=G\left(\bar{u}_{\nu}\right)$ and $\bar{y}=G(\bar{u})$.

Proof We use the condition (4.33) or (4.38) and the fact that $J_{\nu}\left(\bar{u}_{\nu}\right) \leq J_{\nu}(\bar{u})$. Then we get

$$
\begin{aligned}
J(\bar{u}) & +\frac{\delta}{2}\left\{\left\|y_{\nu}-\bar{y}\right\|_{L^{2}(Q)}^{2}+\gamma\left\|y_{\nu}(T)-\bar{y}(T)\right\|_{L^{2}(\Omega)}^{2}\right\}+\frac{\nu}{2}\left\|\bar{u}_{\nu}\right\|_{L^{2}(Q)}^{2} \\
& \leq J\left(\bar{u}_{\nu}\right)+\frac{\nu}{2}\left\|\bar{u}_{\nu}\right\|_{L^{2}(Q)}^{2}=J_{\nu}\left(\bar{u}_{\nu}\right) \leq J_{\nu}(\bar{u})=J(\bar{u})+\frac{\nu}{2}\|\bar{u}\|_{L^{2}(Q)}^{2} .
\end{aligned}
$$

From here, we deduce that $\left\|\bar{u}_{\nu}\right\|_{L^{2}(Q)} \leq\|\bar{u}\|_{L^{2}(Q)}$ and furthermore

$$
\begin{aligned}
& \frac{\delta}{2}\left\{\left\|y_{\nu}-\bar{y}\right\|_{L^{2}(Q)}^{2}+\gamma\left\|y_{\nu}(T)-\bar{y}(T)\right\|_{L^{2}(\Omega)}^{2}\right\} \\
& \quad \leq \frac{\nu}{2}\left(\|\bar{u}\|_{L^{2}(Q)}^{2}-\left\|\bar{u}_{\nu}\right\|_{L^{2}(Q)}^{2}\right) \leq \nu\|\bar{u}\|_{L^{2}(Q)}\left\|\bar{u}-\bar{u}_{\nu}\right\|_{L^{2}(Q)}
\end{aligned}
$$


Now we obtain the estimate

$$
\frac{1}{\sqrt{\nu}}\left\{\left\|y_{\nu}-\bar{y}\right\|_{L^{2}(Q)}+\gamma\left\|y_{\nu}(T)-\bar{y}(T)\right\|_{L^{2}(\Omega)}\right\} \leq\left(\frac{2}{\delta}\|\bar{u}\|_{L^{2}(Q)}\left\|\bar{u}-\bar{u}_{\nu}\right\|_{L^{2}(Q)}\right)^{1 / 2},
$$

where the right hand side converges to zero.

It might be surprising that both of the coercivity conditions (4.12) or (4.34) can be used this time. The reason is that, since $\bar{u}$ and $\bar{u}_{\nu}$ are requested to be as in Theorem 13, here the strong convergence $\bar{u}_{\nu} \rightarrow \bar{u}$ is assumed while Theorem 11 was based only on weak convergence of $\left\{\bar{u}_{\nu}\right\}$.

In [17] the reader can find a deeper analysis of the stability when the Tikhonov parameter $\nu$ goes to zero in the case of a control problem of a linear elliptic partial differential equations. The extension of their results to the case of nonlinear partial differential equations is an open issue.

Acknowledgements The first author was partially supported by Spanish Ministerio de Economía y Competitividad under projects MTM2011-22711 and MTM2014-57531-P. The second is supported by DFG in the framework of the Collaborative Research Center SFB 910, project B6

\section{References}

1. T. Bayen, J. Bonnans, AND F. Silva, Characterization of local quadratic growth for strong minima in the optimal control of semi-linear elliptic equations, Trans. Amer. Math. Soc., 366 (2014), pp. 2063-2087.

2. T. BAYEN AND F. Silva, Second order analysis for strong solutions in the optimal control of parabolic equations, Preprint HAL 2014, https://hal.archives-ouvertes.fr/hal-01096149.

3. F. Bonnans And A. Shapiro, Perturbation Analysis of Optimization Problems, SpringerVerlag, Berlin, 2012.

4. R. Buchholz, H. Engel, E. Kammann, and F. Tröltzsch, On the optimal control of the Schlögl model, Computational Optimization and Applications, 56 (2013), pp. 153-185.

5. E. CASAS, Pontryagin's principle for state-constrained boundary control problems of semilinear parabolic equations, SIAM J. Control Optim., 35 (1997), pp. 1297-1327.

6. E. CASAS, Second order analysis for bang-bang control problems of PDEs, SIAM J. Control Optim., 50 (2012), pp. 2355-2372.

7. E. Casas And K. Chrysafinos, Error Estimates for the Approximation of the Velocity Tracking Problem with Bang-Bang Controls, Submitted. (2015).

8. E. Casas, R. Herzog, and G. WaChSmuth, Optimality conditions and error analysis of semilinear elliptic control problems with $L^{1}$ cost functional, SIAM J. Optim., 22 (2012), pp. $795-820$.

9. E. Casas, C. Ryll, And F. Tröltzsch, Sparse optimal control of the Schlögl and FitzHugh-Nagumo systems, Computational Methods in Applied Mathematics, 13 (2014), pp. $415-442$

10. E. CASAs, C. RYll, And F. Tröltzsch, Second order and stability analysis for optimal sparse control of the FitzHugh-Nagumo equation, SIAM J. Control Optim., 53 (2015), pp. 2168-2202.

11. E. Casas and F. Tröltzsch, Second Order Analysis for Optimal Control Problems: Improving Results Expected from from Abstract Theory, SIAM J. Optim., 22 (2012), pp. 261279.

12. E. Casas and F. Tröltzsch, Second order optimality conditions and their role in PDE control, Jahresbericht der Deutschen Mathematiker-Vereinigung, 117 (2015), pp. 3-44.

13. E. Casas, F. Tröltzsch, and A. Unger, Second order sufficient optimality conditions for a nonlinear elliptic control problem, Z. für Analysis und ihre Anwendungen (ZAA), 15 (1996), pp. 687-707. 
14. J. DunN, On second order sufficient optimality conditions for structured nonlinear programs in infinite-dimensional function spaces, in Mathematical Programming with Data Perturbations, A. Fiacco, ed., Marcel Dekker, 1998, pp. 83-107.

15. A.D. Ioffe and V.M. Tihomirov. Theory of extremal problems. North-Holland Publishing Co., Amsterdam, 1979

16. F. Tröltzsch, Optimal Control of Partial Differential Equations. Theory, Methods and Applications, vol. 112, American Math. Society, Providence, 2010.

17. G. Wachsmuth and D. Wachsmuth, Convergence and regularisation results for optimal control problems with sparsity functional, ESAIM Control Optim. Calc. Var., 17 (2011), pp. 858-886.

18. E. ZEIDLER, Nonlinear functional analysis and its applications. III, Springer-Verlag, New York, 1985. Variational methods and optimization, Translated from the German by Leo F. Boron.

19. E. ZeIDLER, Applied Functional Analysis and its Applications. Main Principles and their Applications, Springer-Verlag, New York, 1995. 